\title{
A Review of Carbon Nanotubes-Based Gas Sensors
}

\author{
Yun Wang and John T. W. Yeow \\ Systems Design Engineering, University of Waterloo, 200 University Avenue West, Waterloo, Ontario, Canada N2L 3G1 \\ Correspondence should be addressed to John T. W. Yeow, jyeow@engmail.uwaterloo.ca
}

Received 31 December 2008; Revised 7 April 2009; Accepted 15 May 2009

Recommended by Michele Penza

\begin{abstract}
Gas sensors have attracted intensive research interest due to the demand of sensitive, fast response, and stable sensors for industry, environmental monitoring, biomedicine, and so forth. The development of nanotechnology has created huge potential to build highly sensitive, low cost, portable sensors with low power consumption. The extremely high surface-to-volume ratio and hollow structure of nanomaterials is ideal for the adsorption of gas molecules. Particularly, the advent of carbon nanotubes (CNTs) has fuelled the inventions of gas sensors that exploit CNTs' unique geometry, morphology, and material properties. Upon exposure to certain gases, the changes in CNTs' properties can be detected by various methods. Therefore, CNTs-based gas sensors and their mechanisms have been widely studied recently. In this paper, a broad but yet in-depth survey of current CNTs-based gas sensing technology is presented. Both experimental works and theoretical simulations are reviewed. The design, fabrication, and the sensing mechanisms of the CNTs-based gas sensors are discussed. The challenges and perspectives of the research are also addressed in this review.
\end{abstract}

Copyright ( $\odot 2009$ Y. Wang and J. T. W. Yeow. This is an open access article distributed under the Creative Commons Attribution License, which permits unrestricted use, distribution, and reproduction in any medium, provided the original work is properly cited.

\section{Introduction}

Gas sensors, or chemical sensors, are attracting tremendous interest because of their widespread applications in industry, environmental monitoring, space exploration, biomedicine, and pharmaceutics. Gas sensors with high sensitivity and selectivity are required for leakage detections of explosive gases such as hydrogen, and for real-time detections of toxic or pathogenic gases in industries. There is also a strong demand for the ability to monitor and control our ambient environment, especially with the increasing concern of the globe warming. Researchers from the national aeronautics and space administration (NASA) are seeking the use of high-performance gas sensors for the identification of atmospheric components of various planets. In addition, nerve agent sensing for homeland security is also at the centre of public concern [1].

Generally, there are several basic criteria for good and efficient gas sensing systems: (i) high sensitivity and selectivity; (ii) fast response time and recovery time; (iii) low analyst consumption; (iv) low operating temperature and temperature independence; (v) stability in performances. Commonly used gas sensing materials include vapour-sensitive polymers, semiconductor metal oxides, and other porous structured materials such as porous silicon [2-4]. Since the most common gas sensing principle is the adsorption and desorption of gas molecules on sensing materials, it is quite understandable that by increasing the contact interfaces between the analytes and sensing materials, the sensitivity can be significantly enhanced.

Recent development of nanotechnology has created huge potential to build highly sensitive, low cost, portable sensors with low power consumption. The extremely high surfaceto-volume ratio and hollow structure of nanomaterials is ideal for gas molecules adsorption and storage. Therefore, gas sensors based on nanomaterials, such as carbon nanotubes (CNTs), nanowires, nanofibers, and nanoparticles, have been investigated widely.

Carbon nanotubes, since been firstly discovered by Iijima in 1991 [5], have drawn the most research interests because of their unique geometry, morphology, and properties. Their preparation, properties (such as electronic, mechanical, thermal, and optical properties), and applications on various fields are all studied intensely. Theoretical and simulation works have also been conducted to understand this nanoscaled material and related phenomenon [6]. 
Carbon nanotubes belong to the family of fullerene structures. There are two types of nanotubes: single-walled carbon nanotubes (SWCNTs) and multiwalled carbon nanotubes (MWCNTs). An SWCNT can be considered as a one-atomthick layer of graphite rolled up into a seamless cylinder with a diameter of several nanometers, and length on the order of 1-100 microns [7]. MWCNTs consist of multiple layers of graphite wrapped up together to form a tube shape, sharing the same central axis. The structure of carbon nanotubes provides them with inherently unique electrical, physical, and chemical properties. Mechanically, CNTs are the strongest and stiffest fibers that are known currently due to the $\mathrm{C}-\mathrm{C}$ bond. Thermally, $\mathrm{CNTs}$ have high thermal stability in both vacuum and air. In terms of electrical properties, CNTs can be either metallic or semiconducting, depending upon the tube diameter and the chirality (the direction in which the graphite sheet is rolled to form the tube) [8]. The chirality is usually represented by an integer pair $(n, m)$. Nanotubes with $n-m=3 j$ ( $j$ being a nonzero integer) are metallic while all the others are semiconducting. The dielectric property of nanotubes is highly anisotropic due to their nearly one-dimensional structures, which may enable nanotubes to carry high currents with negligible heating effect [9].

Upon exposure to certain gases, the change in the properties of CNTs or CNTs-based composites can be detected by various methods. As a result, CNTs-based gas sensing systems and the theoretical analyses of gas adsorption and collision effects on the nanotubes have been the subjects of intense research $[10,11]$. In this paper, the current status of CNTs gas sensor-related technologies is reviewed. This paper is organized as follow: in Section 2 the synthesis techniques of CNTs and different fabrication methods of CNTs-based gas sensors will be introduced; Section 3 will be focussed on the simulation and theoretical works on the interaction between CNTs and the adsorbed gas molecules; in Section 4, CNTs gas sensors based on different sensing mechanisms will be summarized with their design and sensing behaviour; Section 5 will highlight the functionalization of CNTs and CNTs-based nanocomposites for gas sensing; the conclusion with the chance and challenges that the CNTs gas sensing technology is facing will be addressed in Section 6.

\section{Fabrication of CNTs Gas Sensors}

2.1. Growth of CNTs. Three main techniques to prepare CNTs are as follows: (i) arc-discharge technique; (ii) laser ablation technique; (iii) chemical vapour deposition (CVD) technique [12].

The carbon arc-discharge method is the first technique that was used to grow CNTs. The process is carried out in a vacuum chamber with two carbon electrodes as carbon source. Inert gas (typically helium) is supplied to increase the speed of carbon deposition. When high dc voltage is applied between the carbon anode and cathode, plasma of the inert gas is generated to evaporate the carbon atoms. The ejected carbon atoms are then deposited on the negative electrode to form CNTs. Both SWNTs and MWNTs can be grown by this method, while the growth of SWNTs requires catalysts. It is the principal method to produce highquality CNTs with nearly perfect structures. In the laser ablation technique, a carbon target is ablated by intense laser pulses in a furnace in the presence of an inert gas and a catalyst. CNTs are formed and collected on a cold substrate. Both the arc-discharge and laser-ablation methods require high growth temperature, which is about $3000-4000^{\circ} \mathrm{C}$ for the evaporation of carbon atoms from solid carbon source.

In a CVD system, a gas hydrocarbon source (usually methane, acetylene or ethylene) flows into the reaction chamber. The hydrocarbon molecules are broken into reactive species at the temperature range of $550-1000^{\circ} \mathrm{C}$. The reactive species react in the presence of catalysts (usually metal particles such as $\mathrm{Ni}, \mathrm{Fe}$ or $\mathrm{Co}$ ) that are coated on the substrate, leading to the formation of CNTs. Compared with the first two techniques; CNTs can be synthesized at relatively low temperature using CVD method. Therefore, this technique is more efficient and allows scaleup growth of SWCNTs. By modification and calculated control of the growth parameters, vertically aligned MWCNTs growth can be achieved by CVD technique. This enhances CNTs electronic properties in different applications. High-quality SWCNTs can also be obtained by the optimization of the catalysts. However, one of the main disadvantages of CVD technique is the relatively high defect densities in MWCNTs, which can be attributed to the lack of sufficient thermal energy.

In all of these three growth methods, CNTs come with a number of impurities, which may have negative effects on CNTs' inherent properties. In order to make CNT-based devices more efficient and consistent, purification is an important process to obtain high-quality nanotubes. Generally, the most commonly observed impurities are carbonaceous impurities and metallic impurities. The carbonaceous components are the byproducts of the reaction process while the metallic impurities are residual catalysts. To eliminate the carbonaceous impurities, the main method is oxidation. Two approaches are developed: (i) gas phase purification, which involves high temperature and has low yield production; (ii) liquid phase purification, where nanotubes are washed in acidic solutions. Nitric acid, sulphuric acid, and their mixture are all commonly used for liquid purification [13]. Metallic impurities can be removed by heating the samples up to the evaporation temperature of the impurity. With the development and modification of different purification procedure, so far CNTs samples with a purity of up to $99.6 \%$ can be obtained [14]. The quality of as-grown CNTs and the purification can be inspected by microscopic techniques such as atomic force and scanning tunneling microscopy, or spectroscopic techniques such as Raman spectroscopy.

2.2. Fabrication of CNTs Gas Sensors. There are several methods to integrate CNTs to different gas sensor structures. Li et al. developed a resistive gas sensor by simply casting SWCNTs on interdigitated electrodes (IDEs) [14]. The electrodes were fabricated by photolithography and evaporation of $\mathrm{Ti}$ and $\mathrm{Au}$ (60 nm in thickness together) on silicon oxide. Asgrown SWCNTs were purified with acid first and then by air 


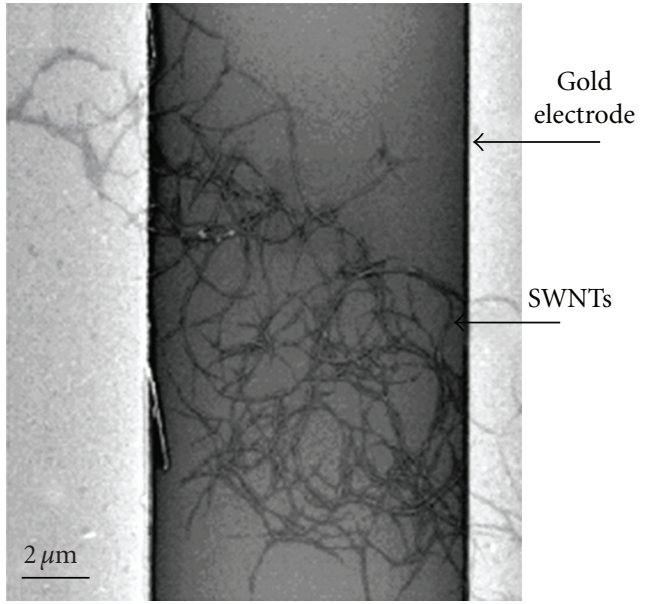

FIgURE 1: SEM image of SWCNTs across two gold electrodes [14].

oxidation before being integrated with the IDEs. As a result, the final SWCNTs had a relatively high purity up to $99.6 \%$, and the impact of impurities on the sensor's characteristic was minimized. The purified nanotubes were then dispersed in dimethylformamide (DMF) and drop-deposited onto the electrode area. A network of nanotubes subsequently formed after the evaporation of DMF, as shown in Figure 1. Another simply method is screen-printing of CNTs onto patterned electrodes. Lee et al. [15] screen-printed CNTs paste mixed with MWNTs, terpineol, ethylcellulose, and glass frits onto electrode-coated glass as the gas sensing element for $\mathrm{NO}_{2}$ detection. The organic binder can be removed by annealing in $\mathrm{N}_{2}$ ambient.

Fabrication of CNTs sensors can also be achieved by dielectrophoresis (DEP) method. DEP is the electrokinetic motion of dielectrically polarized materials in nonuniform electric fields and has been used to manipulate CNTs for separation, orientation, and positioning of CNTs [16-22]. Suehiro et al. [23] demonstrated that the DEP fabrication could establish a good electrical connection between CNTs and the electrodes. During fabrication, the CNTs with high purity were suspended in ethanol and ultrasonicated for 60 minutes. The system to fabricate the CNTs-based gas sensor is schematically depicted in Figure 2. An interdigitated microelectrode was patterned on a glass substrate. The electrode had a castle-wall pattern in order to form high and low electric field regions periodically. The castle-wall electrode was surrounded by a silicon rubber spacer to form a sealed chamber in which CNTs suspension was continuously fed from a reservoir by a peristaltic pump. The DEP trapping of MWCNTs on the microelectrode was performed with an ac voltage. After a desired period of time, the DEP process was stopped and the ethanol was evaporated at room temperature. The DEP-fabricated CNTs gas sensors successfully detected various vapours such as $\mathrm{NH}_{3}, \mathrm{NO}_{2}$, $\mathrm{SO}_{2}$, and $\mathrm{HF}$. With this technique, the amount of trapped CNTs can be controlled by monitoring electrical impedance of the sensor and various metal materials can be employed as the electrodes [24].

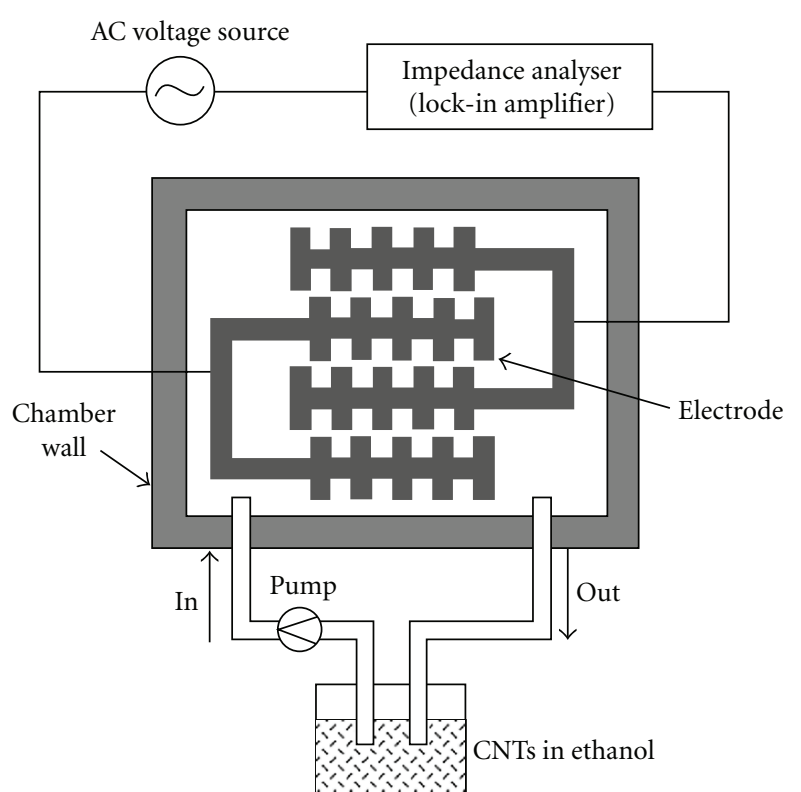

FIGURE 2: Schematic diagram of the experimental setup for MWCNT gas sensor fabrication on a microelectrode using DEP [23].

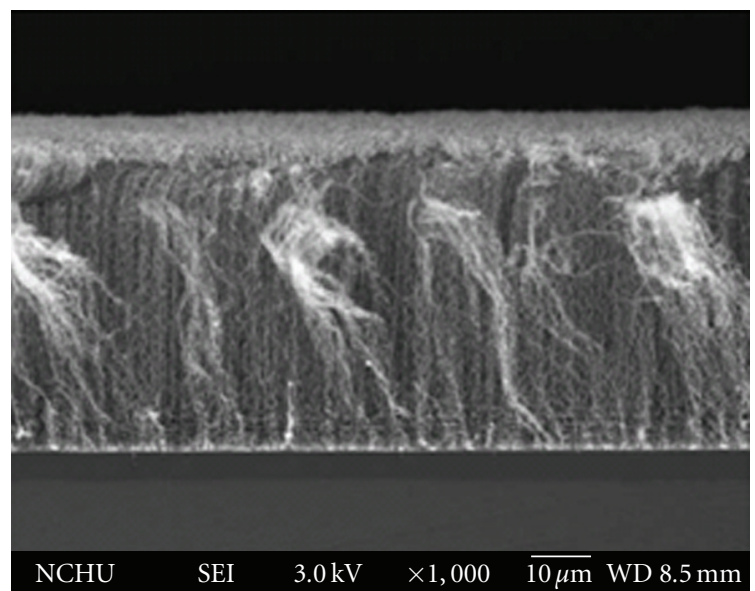

FIGURE 3: Cross-sectional SEM images of the vertically aligned CNTs mat [25].

To obtain well aligned CNTs for better sensor behaviour, directly growth of the CNTs mats on the sensor substrates is required. Huang et al. [25] fabricated a three terminal $\mathrm{N}_{2}$ gas sensor with vertically aligned CNTs. The CNTs were grown by thermal CVD on (100) n-type silicon substrate at $700^{\circ} \mathrm{C}$. A $30 \mathrm{~nm}$ thick Fe layer was sputtered on the substrate as catalyst and $\mathrm{C}_{2} \mathrm{H}_{2}$ was used as the carbon source. A preheating process was carried out before the growth of CNTs and the substrate was cooled down at room temperature in $\mathrm{N}_{2}$ ambient. The as-grown CNTs mat is shown in Figure 3.

Jang et al. [26] fabricated an $\mathrm{NH}_{3}$ gas sensor with laterally aligned MWNTs. N-type heavily doped Si wafer with $\mathrm{SiO} 2$ layer on top was used as the substrate. The $\mathrm{Nb}$ electrodes 


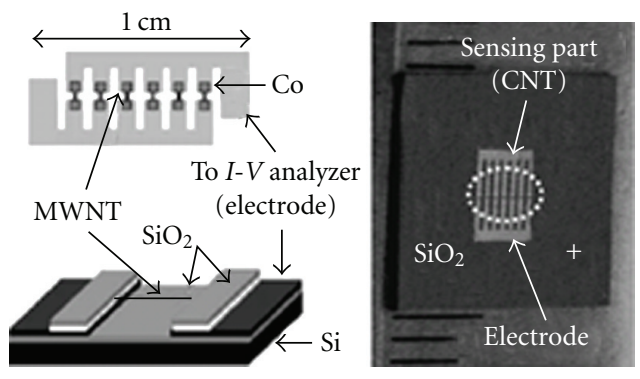

(a)

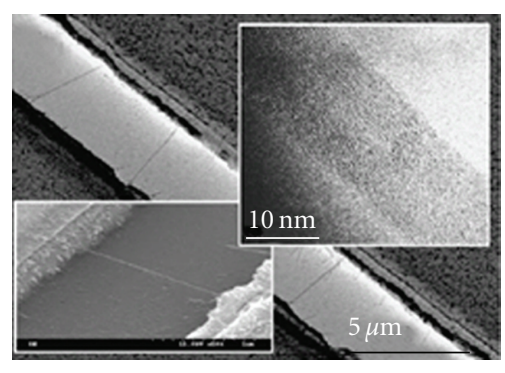

(b)

Figure 4: (a) Photograph of completed MWNTs based chemical sensor, (b) SEM image of aligned CNTs resting on $\mathrm{SiO}_{2}$ surface after directed growth. The CNTs show clear alignment in the direction of the electric field. The insert shows TEM image of laterally grown CNTs [26].

were deposited and patterned on the substrate. The catalyst and oxide were then deposited and patterned by lift-off to be aligned to the electrodes, where top oxide layer is introduced as a barrier layer for vertical growth and exposure gas, covering the top of the Co catalytic layer. Aligned MWNTs across the gap between the electrodes were grown in thermal CVD system with electrical feed-through. Figure 4 shows the structure of the sensor and the laterally aligned CNTs connecting the electrodes.

Tabib-Azar and Xie [28] also successfully prepared selfaligned and self-welded MWNTs between prepatterned electrodes by low pressure CVD (LPCVD) for $\mathrm{NH}_{3} \mathrm{OH}$ and $\mathrm{HCI}$ sensing. Ding et al. [29] achieved the growth of aligned CNTs in an anodic aluminum oxide (AAO) template for hydrogen sensor. With the development in the preparation techniques, controllable fabrication of CNTs-based gas sensors with improved performance can be achieved [30].

\section{Theoretic and Simulation Studies of the Gas Adsorption on CNTs}

3.1. Molecule Adsorption on SWNTs. The adsorption of various gas molecules on SWNTs is usually studied by first-principles calculations using density functional theory (DFT). The binding energy, tube-molecule distance, and change transfers are generally investigated. Peng and Cho [31] studied the adsorption of $\mathrm{NO}_{2}$ on to SWNTs by this method. Figure 5 shows one binding configuration for $\mathrm{NO}_{2}$ gas molecule on the $(10,0)$ SWNT with three units. $\mathrm{NO}_{2}$

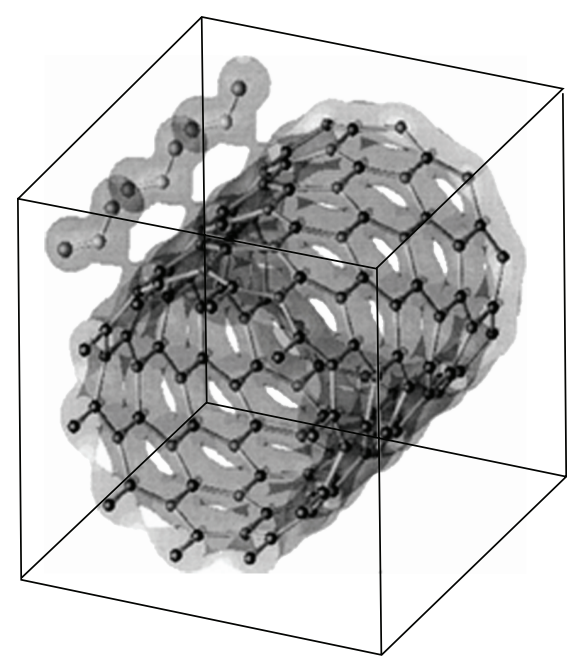

(a)

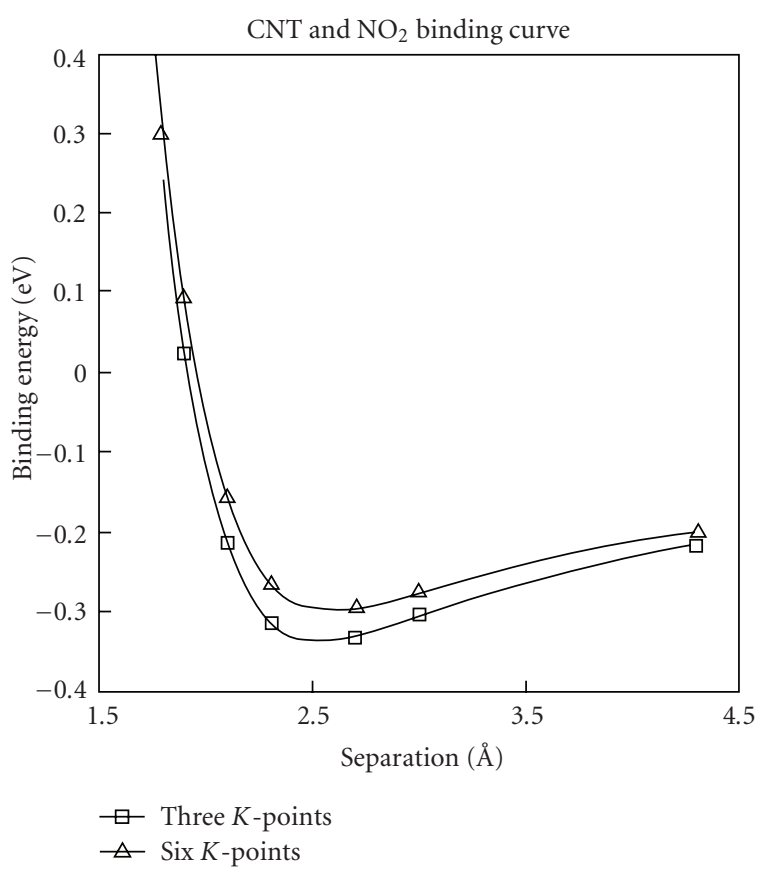

(b)

FIgure 5: (a) Total valence electron charge density plot. The value of charge contour is $0.0015\left(\mathrm{el} / \AA^{3}\right)$ showing the binding charge between the SWNT $(10,0)$ and the $\mathrm{NO}_{2}$ molecule. Three units are shown in this figure. (b) Binding energy curve for $\mathrm{NO}_{2}$ interacting with $(10,0)$ SWNT as a function of distance from $\mathrm{NO}_{2}$ to the nanotube. The solid line curve is a fitting with universal binding curve [31].

gas molecule of this configuration is found to bind with SWNT with adsorption energy of $0.3 \mathrm{eV}$, and it is also found that the molecule has high diffusion kinetics on nanotubes surfaces. Electron density analysis shows that charge transfer is induced from $\mathrm{C}$ atom to the $\mathrm{NO}_{2}$ gas molecule leading to hole (or p-type) doping of semiconducting $(10,0)$ nanotubes. 
TABle 1: Equilibrium tube-molecule distance $(d)$, adsorption energy $\left(E_{a}\right)$, and charge transfer $(Q)$ of various molecules on $(10,0),(17,0)$, and $(5,5)$ individual SWNTs ${ }^{\mathrm{a}}$. The optimal adsorption sites are given in the table: T (top of a carbon atom), B (top of the centre of the C-C bond), C (top of the centre of carbon hexagon) [27]. (a) Tube-molecule distance $d$ is defined as the nearest distance between atoms on the molecule and the nanotube for $\mathrm{T}$ site, or the distance between the centre of the gas molecule and the centre of the carbon hexagon (carboncarbon bond) for the $\mathrm{C}(\mathrm{B})$ site. The adsorption energy $E_{a}(d)$ is defined as the total energy gained by molecule adsorption at equilibrium distance: $E_{a}(d)=E_{\text {tot }}($ tube + molecule $)-E_{\text {tot }}($ tube $)-E_{\text {tot }}$ (molecule). Charge transfer $(Q)$ denotes the total Mulliken charge number on the molecules, positive $(Q)$ means charge transfer from molecule to tube.

\begin{tabular}{lccccccccc}
\hline & $\mathrm{NO}_{2}$ & $\mathrm{O}_{2}$ & $\mathrm{H}_{2} \mathrm{O}$ & $\mathrm{NH}_{3}$ & $\mathrm{CH}_{4}$ & $\mathrm{CO}_{2}$ & $\mathrm{H}_{2}$ & $\mathrm{~N}_{2}$ & $\mathrm{Ar}$ \\
\hline$(10,0)$ SWNT & & & & & & & & & \\
\hline$d(\AA)$ & 1.93 & 2.32 & 2.69 & 2.99 & 3.17 & 3.20 & 2.81 & 3.23 & 3.32 \\
$E_{a}(\mathrm{meV})$ & 797 & 509 & 143 & 149 & 190 & 97 & 113 & 164 & 57 \\
$Q(\mathrm{e})$ & -0.061 & -0.128 & 0.035 & 0.031 & 0.027 & 0.016 & 0.014 & 0.008 & 0.01 \\
Site & $\mathrm{T}$ & $\mathrm{B}$ & $\mathrm{T}$ & $\mathrm{T}$ & $\mathrm{C}$ & $\mathrm{C}$ & $\mathrm{C}$ & $\mathrm{C}$ & $\mathrm{C}$ \\
\hline$(5,5)$ SWNT & & & & & & & & & \\
\hline$d(\AA)$ & 2.16 & 2.46 & 2.68 & 2.99 & 3.33 & 3.54 & 3.19 & 3.23 & 3.58 \\
$E_{a}(\mathrm{meV})$ & 427 & 306 & 128 & 162 & 122 & 109 & 84 & 123 & 82 \\
$Q(\mathrm{e})$ & -0.071 & -0.142 & -0.033 & 0.033 & 0.022 & 0.014 & 0.016 & 0.011 & 0.011 \\
Site & $\mathrm{T}$ & $\mathrm{B}$ & $\mathrm{T}$ & $\mathrm{T}$ & $\mathrm{C}$ & $\mathrm{C}$ & $\mathrm{C}$ & $\mathrm{C}$ & $\mathrm{C}$ \\
\hline$(17,0)$ SWNT & & & & & & & & & \\
\hline$d(\AA)$ & 2.07 & 2.50 & 2.69 & 3.00 & 3.19 & 3.23 & 2.55 & 3.13 & 3.34 \\
$E_{a}(\mathrm{meV})$ & 687 & 487 & 127 & 133 & 72 & 89 & 49 & 157 & 82 \\
$Q(\mathrm{e})$ & -0.089 & -0.096 & 0.033 & 0.027 & 0.025 & 0.015 & 0.012 & 0.006 & 0.01 \\
Site & $\mathrm{T}$ & $\mathrm{B}$ & $\mathrm{T}$ & $\mathrm{T}$ & $\mathrm{C}$ & $\mathrm{C}$ & $\mathrm{C}$ & $\mathrm{C}$ & $\mathrm{C}$ \\
\hline
\end{tabular}

Zhao et al. [27] studied the adsorption of various gas molecules $\left(\mathrm{NO}_{2}, \mathrm{O}_{2}, \mathrm{NH}_{3}, \mathrm{~N}_{2}, \mathrm{CO}_{2}, \mathrm{CH}_{4}, \mathrm{H}_{2} \mathrm{O}, \mathrm{H}_{2}\right.$, Ar) on both single SWNT and SWNT bundles using first principles method. The self-consistent field (SCF) electronic structure calculations are performed based on density functional theory (DFT) with either localized basis (DMol) or planewave basis (CASTEP).

For individual SWNT, the equilibrium tube-molecule distance, adsorption energy, and change transfer for various molecules on $(10,0),(17,0)$ and $(5,5)$ SWNTs were calculated, as shown in Table 1. The results show that most of the studied molecules (except for $\mathrm{NO}_{2}$ and $\mathrm{O}_{2}$ ) are charge donors with small charge transfer $(0.01 \sim 0.035 \mathrm{e})$ and weak binding $(\leq 0.2 \mathrm{eV})$. These gas molecules can be identified as physisorption. For $\mathrm{O}_{2}$ and $\mathrm{NO}_{2}$, it shows that they both are charge acceptors with large charge transfer and adsorption energies. These results are consistent with reported experimental results [32,33]. It also demonstrated that there is no clear dependence of adsorption on the tube size and chirality.

For SWCNTs bundle, there are four distinct sites for gas molecules to be adsorbed onto (shown in Figure 6): (i) the external surface of the bundle; (ii) the groove formed at the contact between adjacent tubes on the outside of the bundle; (iii) the interior pore of individual tubes; (iv) interstitial channel formed between three adjacent tubes within the bundle $[34,35]$. The gas adsorption on these sites is decided by the binding energy of the gas molecule as well as the site availability. Some of these sites may not be available for certain gases because of the gas molecule dimension and the site diameter. The interior pore is only accessible when the SWCNT is uncapped or has defects on the tube

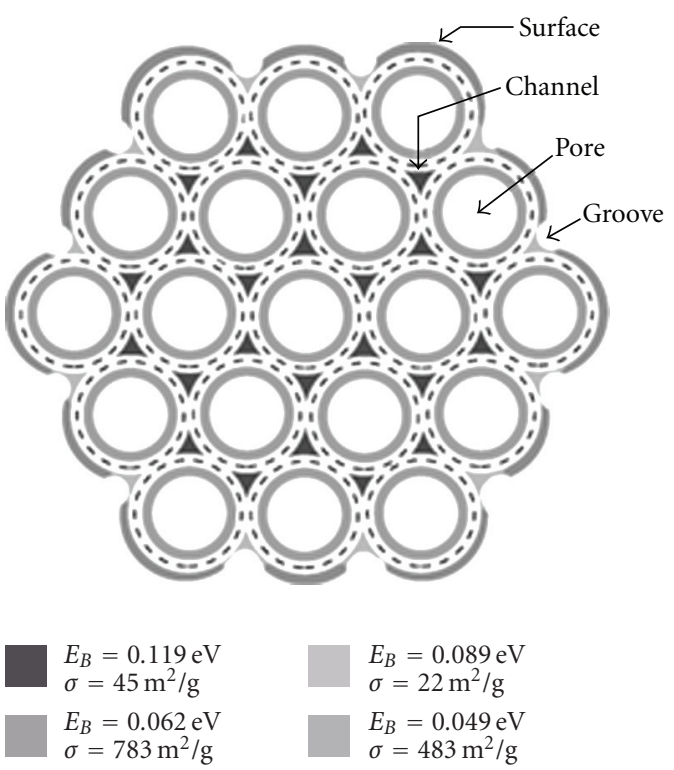

FIgure 6: Schematic structure of a SWNT bundle showing the available sites for gas adsorption. Dashed line indicates the nuclear skeleton of the nanotubes. Binding energies $\left(E_{B}\right)$ and specific surface area contributions $(\sigma)$ for $\mathrm{H}_{2}$ adsorption on these sites are indicated [34].

walls [36]. Williams and Eklund [34] calculated the binding energy $\left(E_{B}\right)$ and specific surface area contributions $(\sigma)$ for $\mathrm{H}_{2}$ adsorption on these sites as shown in Figure 6. It shows that $E_{B}$ (channels) $>E_{B}$ (grooves) $>E_{B}$ (pores) $>E_{B}$ (surface), while the pores and surface have much larger specific surface 
TABle 2: Equilibrium tube-molecule distance $(d)$, adsorption energy $\left(E_{a}\right)$ and charge transfer $(Q)$ of the $\mathrm{H}_{2}$ molecule on different adsorption sites in the $(10,10)$ SWNT bundle [27].

\begin{tabular}{lccc}
\hline Site & $d(\AA)$ & $E_{a}(\mathrm{meV})$ & $Q(\mathrm{e})$ \\
\hline Surface & 3.01 & 94 & 0.014 \\
Pore & 2.83 & 111 & 0.012 \\
Groove & 3.33 & 114 & 0.026 \\
Interstial & 3.33 & 174 & 0.035 \\
\hline
\end{tabular}

area contributions. Zhao et al. [27] also calculated the tubemolecule distance, adsorption energy, and charge transfer of these sites for $\mathrm{H}_{2}$ adsorption using first principles method, as shown in Table 2. These results are consistent with William's results obtained by empirical force field simulation.

3.2. Effects of Molecule Adsorption on Electronic Properties of CNTs. Generally, the electronic property of an SWNT is determined by the tube chirality and diameter [37]. However, it has been demonstrated experimentally that the electronic properties of SWNTs are very sensitive to their chemical environment, especially on oxygen exposure. Collins et al. [33] measured the electrical resistance, thermoelectric power (TEP), and local density of states of SWNTs by transport measurement and scanning tunneling spectroscopy. The results showed that these parameters can be reversibly "tuned" by exposure to very small concentrations of oxygen, as shown in Figure 7. Isolated semiconducting nanotubes can be converted into apparent metals through roomtemperature exposure to oxygen. Sumanasekera et al. [38] also demonstrated experimentally that, by degassing the SWNTs mat in vacuum from air at $500 \mathrm{~K}$, the TEP value decreased slowly from an initial value of $+54 \mu \mathrm{V} / \mathrm{K}$, changed sign, and then eventually approached a constant value of $-44 \mu \mathrm{V} / \mathrm{K}$, as shown in Figure 8. Nuclear magnetic resonance (NMR) study of SWNTs also proved that the spin-lattice relaxation rates of all nanotubes increased dramatically upon exposure to oxygen [39]. Jhi et al. studied the electronic and magnetic properties of oxidized CNTs theoretically using the ab initio pseudopotential total energy method [40]. The calculations showed consistent results with the experimental ones. These results demonstrated the potential for CNTs application as sensitive gas sensors. However, they also indicate that many reported electronic properties measured on as-prepared nanotubes should be assigned to oxygendoped CNTs instead of intrinsic CNTs [33, 38].

Generally, the electronic property changes of CNTs upon exposure to gas molecules are attributed to the charge transfer between the molecules and the nanotubes (the molecules act as electron donors or acceptors). However, Sumanasekera et al. [38] demonstrated experimentally that both of the thermopower $S$ and the resistance of the degassed SWNT mat $R$ can be very sensitive to inert gases $\left(\mathrm{N}_{2}\right.$ and $\mathrm{He}$, which can hardly have electron transfers with CNTs) for $T$ (temperature) $>100 \mathrm{~K}$, as shown in Figure 8. In the case of these gases, it is expected that the charge transfer between the molecules and the nanotubes is negligible. Without charge transfer, it is believed that the resistance changes are due

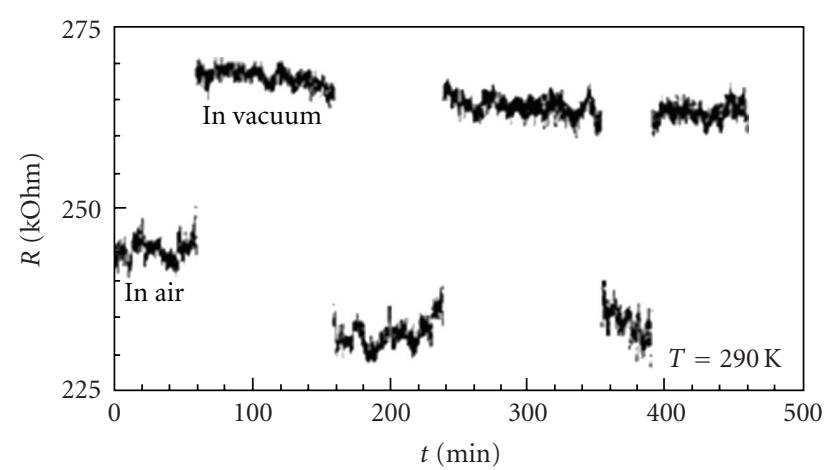

(a)

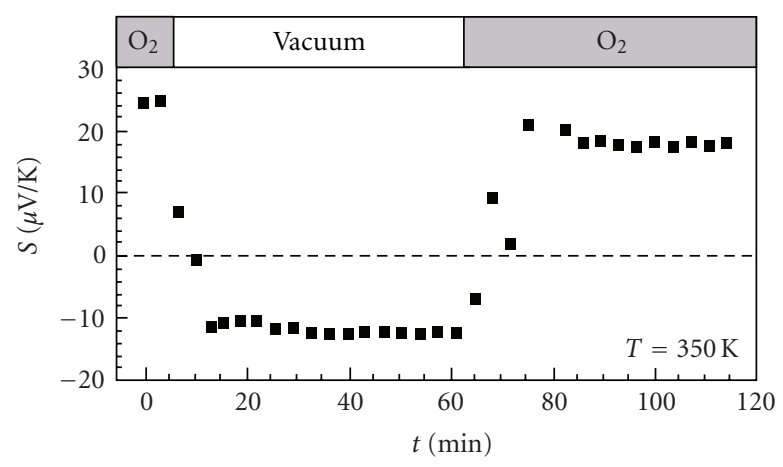

(b)

FIGURE 7: (a) Sensitivity of the electrical resistance $R$ of SWNT films to gas exposure at $290 \mathrm{~K}$; (b) sensitivity to environmental conditions of thermoelectric power $S$ for SWNTs at $350 \mathrm{~K}$. Both the magnitude and the sign can be altered by oxygen exposure. In vacuum, $S$ is ntype, whereas in an oxygen environment, $S$ is p-type, with a larger magnitude [33].

to the change in the electron and hole free carrier lifetimes (or, equivalently, the carrier mobility). These large changes in the carrier lifetime can be caused either by the increased carrier scattering from dynamic defect states associated with momentarily adsorbed gas or due to nonthermal, localized SWNT phonons generated by collisions of the gas molecules with the tube wall.

Based on these observations, it is summarized that the thermoelectric response of a bundle of SWNTs to variety of gases can be either caused by a charge-transfer-induced change of Fermi energy or by the additional scattering channel (identified with impurity sites associated with the adsorbed molecules) for conduction electrons in the tube wall. An equation was developed to understand this assumption [36]:

$$
S=S_{0}+\left(\rho_{a} / \rho_{0}\right)\left(S_{a}-S_{0}\right),
$$

where $S_{0}$ and $S_{a}$ are, respectively, the contributions to the thermopower from the host resistivity $\rho_{0}$ and the additional impurity resistivity $\rho_{a}$ associated with the adsorbed gas. This equation is known as the Nordheim-Gorter $(\mathrm{N}-\mathrm{G})$ relation [41]. When applied to the thermoelectric transport in SWNTs bundles with adsorbed gas molecules, it can indicate 


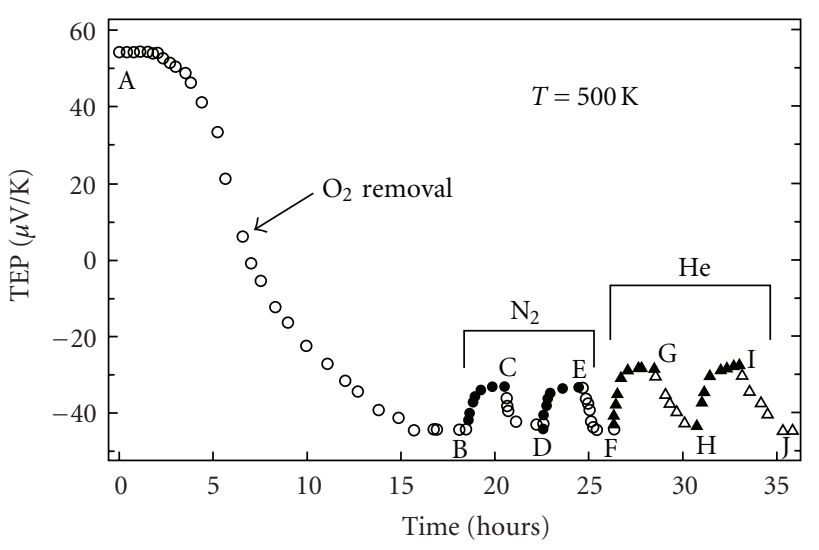

(a)

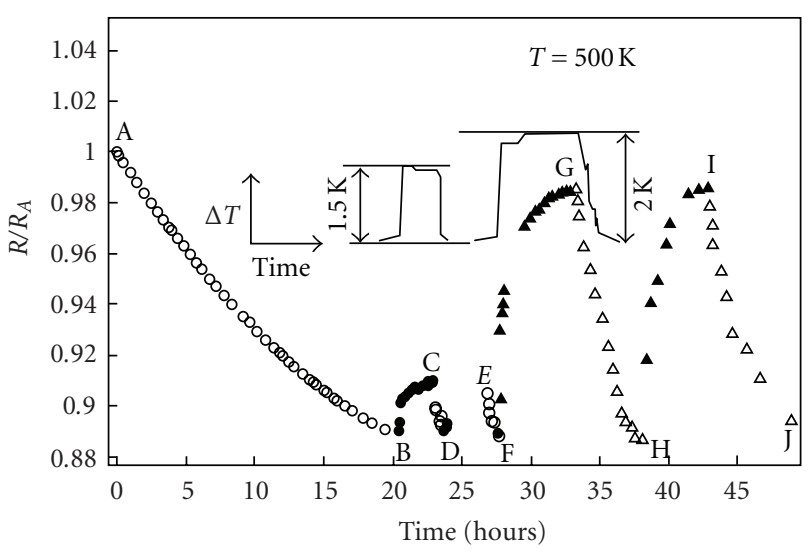

(b)

Figure 8: (a) Thermopower $S$ versus time for a mat of SWNTs at $T=500 \mathrm{~K}$ initially saturated with air at ambient conditions. The sample is under dynamic vacuum when open symbols are used and dark symbols representing intervals when $\mathrm{N}_{2}$ and $\mathrm{He}$ are present. $\mathrm{N}_{2}$ is introduced at $\mathrm{B}$ and $\mathrm{D} ; \mathrm{He}$ is introduced at $\mathrm{F}$ and $\mathrm{H}$. Vacuum pumping is applied at A, C, E, G, and I; (b) four probe resistance ratio of SWNT mat at $500 \mathrm{~K}$ versus time. Data normalized to sample resistance at A. Points A through I have the same meaning as described for (a). Shown in the inset is the time dependence of the temperature during the admission of each gas and subsequent pumping [38].

whether the gas molecules are physisorbed or chemisorbed, as shown in Figure 9.

The effects of other gases such as alcohol and water molecules on the electrical transport of SWNT bundles are also studied [42]. Strong effects on both the TEP and resistivity for methanol, ethanol, isopropanol, and butanol were observed. The N-G plots indicated that these molecules are all physisorbed to the tube wall. However, water vapour does not have any effect on the TEP but has a significant impact on the resistance, as shown in Figure 10.

\subsection{Simulation Study for Functionalization of CNTs Gas} Sensors. Theoretical and simulation research cannot only explain the observed experimental results with in-depth mechanism, but can also simulate the CNTs behaviours

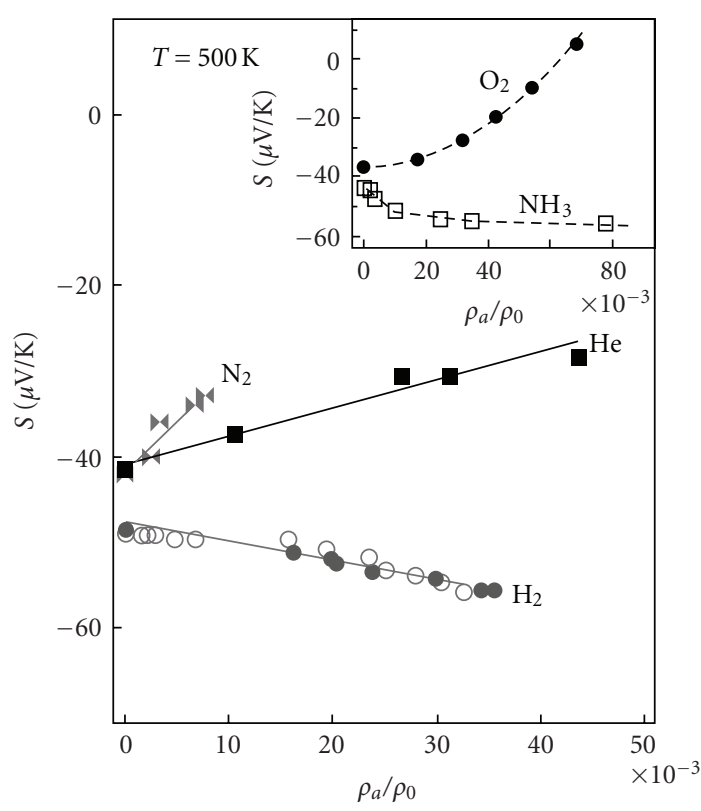

Figure 9: N-G plots ( $S$ versus $\rho$ ) showing the effect of gas adsorption on the electrical properties of the mat. A linear N-G plot indicates that the physisorption is taking place. The inset shows the $\mathrm{N}-\mathrm{G}$ plots for $\mathrm{O}_{2}$ (electron acceptor) and $\mathrm{NH}_{3}$ (electron donor). The data in the inset strongly curved indicating chemisorption is taking place [36].

under certain assumptions of interest before carrying out the actual experiment.

It has been demonstrated that the electronic properties of semiconducting CNTs are very sensitive to certain gas exposure. However, the range of molecules that can be detected by CNTs sensors is limited to the molecules that have large binding energies and charge transfers with the nanotubes. Gas molecules such as toxic gases (CO), water, and bimolecular cannot be detected since they do not adsorb on the nanotubes surface. To overcome this challenge, Peng et al. [43] proposed the design of a new type of CNTs sensors with modified electronic and chemical properties by substitutional doping of impurity atoms (boron and nitrogen) into intrinsic SWNTs or by using composite $\mathrm{B}_{x} \mathrm{C}_{y} \mathrm{~N}_{z}$ nanotubes. To investigate the validation of the idea, the ab initio simulations are performed with the VASP using the density functional theory (DFT) and the local density approximation (LDA) with ultrasoft pseudopotential, planewave basis sets, and periodic boundary conditions.

The interaction of these boron- or nitrogen-doped CNTs with both carbon monoxide and water molecules was calculated. It demonstrated that, when an intrinsic CNT is doped with impurities through a replacement of carbon atoms, the local physical properties around the impurity atoms undergo a significant change, resulting in the change of the local chemical reactivity. The simulation results for water adsorption on both the intrinsic and modified CNTs are shown in Table 3. All the configurations studied are shown in Figure 11. It shows that CNTs with boron doping and intrinsic $\mathrm{BC}_{2} \mathrm{~N}$ type 2 nanotubes have better sensitivity 


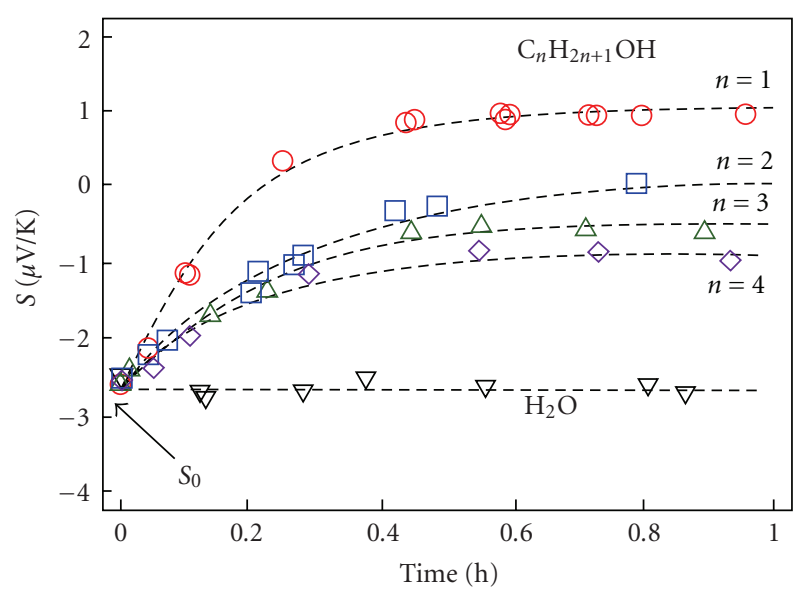

(a)

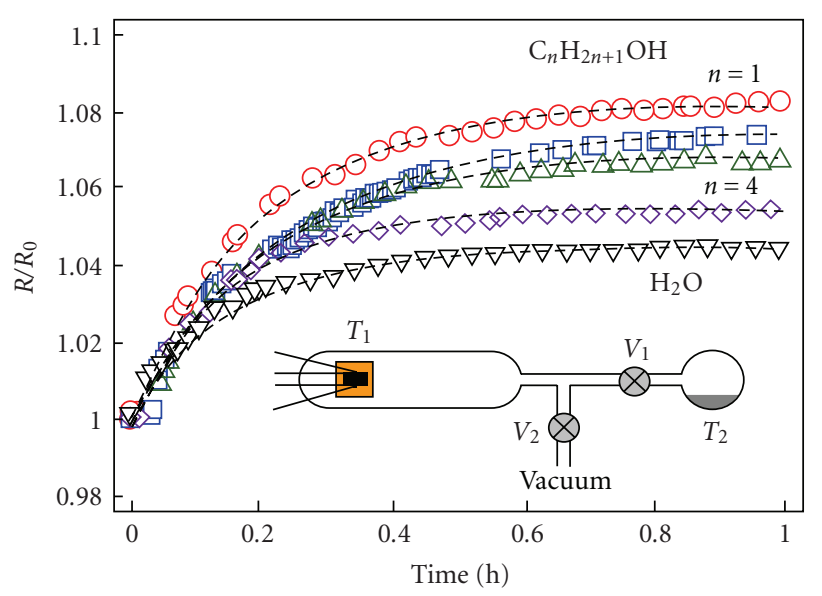

(b)

FIgURE 10: (a) The time dependence of the TEP response of a SWNT thin film to successive exposure to vapours of water and alcohol molecules $\left(\mathrm{C}_{n} \mathrm{H}_{2 n+1} \mathrm{OH} ; n=1-4\right)$ at $40^{\circ} \mathrm{C}$; (b) the time dependence of the normalized four-probe resistance response of an SWNT thin film to successive exposure to vapours of water and alcohol molecules at $40^{\circ} \mathrm{C}$ [42].

to $\mathrm{H}_{2} \mathrm{O}$ due to the strong interaction and a large electron charge transfer. The author also concluded that $\mathrm{CO}$ and $\mathrm{H}_{2} \mathrm{O}$ molecules undergo chemical adsorption with the borondoped CNTs, while physical adsorption with the nitrogendoped CNTs-based on the value of the binding energy. Since the synthesize of $\mathrm{B}$ - or/and $\mathrm{N}$-doped CNTs and $\mathrm{B}_{x} \mathrm{C}_{y} \mathrm{~N}_{z}$ nanotubes have already been proved feasible experimentally $[44,45]$, it is believed that CNTs base gas sensors with a wide range of detectable gas molecules can be achieved experimentally.

\section{Design of CNTs Gas Sensors Based on Different Sensing Mechanism}

4.1. Resistance Change. It has been demonstrated that CNTs electronic properties can be extremely sensitive to the exposed environment due to gas molecule adsorption. The
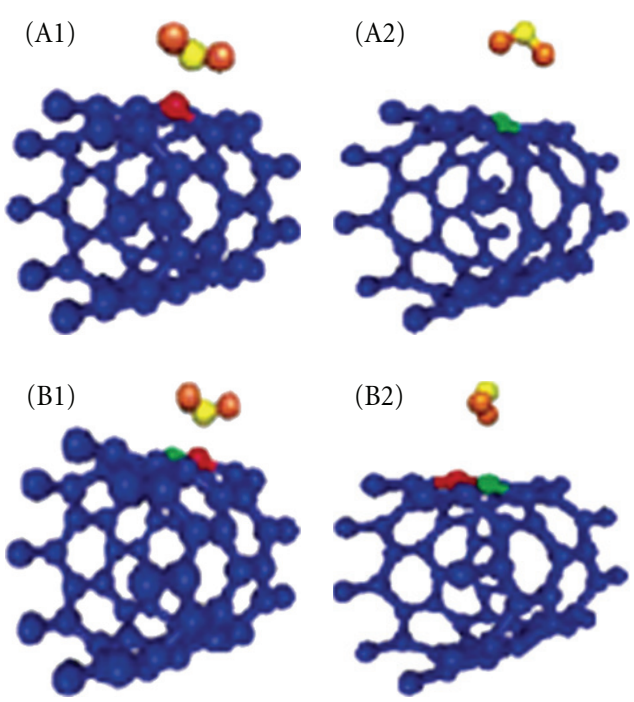

(B2)
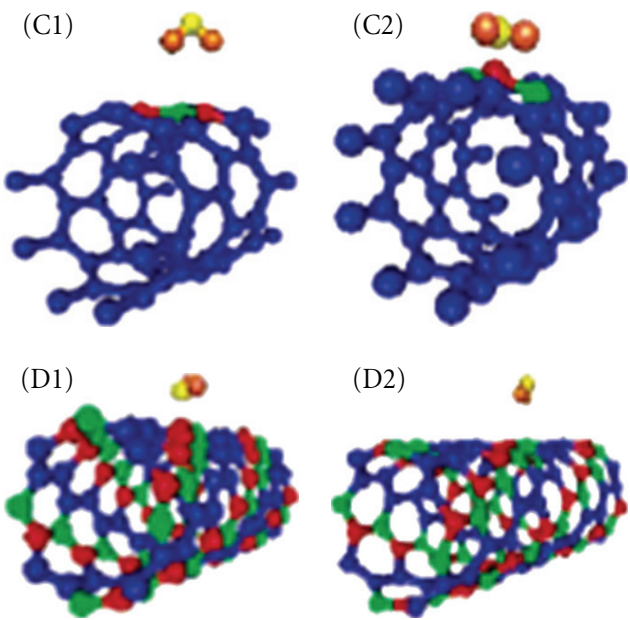

(D2)
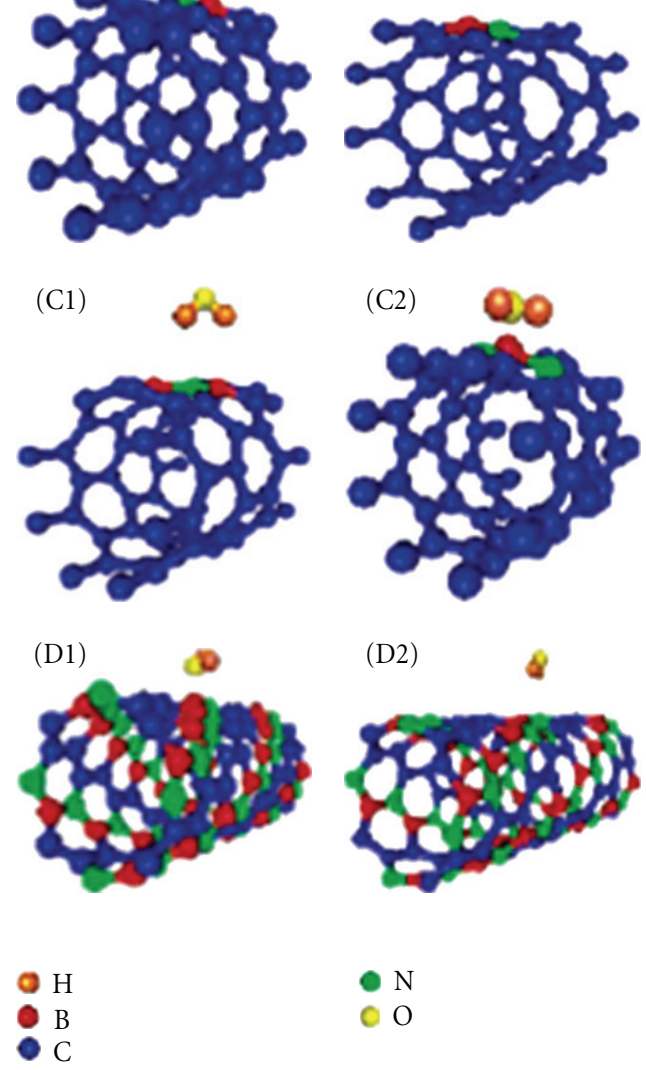

FIGURE 11: Configurations for water adsorption on modified CNTs. (A1) Semiconducting $(8,0)$ CNT with one carbon atom replaced by a boron atom that binds to an $\mathrm{H}_{2} \mathrm{O}$ molecule, where the boron atom is close to the oxygen atom. (A2) Semiconducting $(8,0) \mathrm{CNT}$ with one carbon atom replaced by one Nitrogen atom binds to an $\mathrm{H}_{2} \mathrm{O}$ molecule, where the nitrogen atom is close to the oxygen atom. Semiconducting $(8,0)$ CNT with one carbon atom replaced by a boron atom and another by a nitrogen atom that binds to an $\mathrm{H}_{2} \mathrm{O}$ molecule, where the boron atom is close to the oxygen atom as in (B1), and the nitrogen atom is close to the oxygen atom as in (B2). (C1) Semiconducting $(8,0)$ CNT with two carbon atoms replaced by two boron atoms and one by a nitrogen atom that binds to an $\mathrm{H}_{2} \mathrm{O}$ molecule, where the nitrogen atom is close to the oxygen atom. (C2) Semiconducting $(8,0)$ CNT with one carbon atom replaced by one boron atom and two others by two nitrogen atoms that bind to an $\mathrm{H}_{2} \mathrm{O}$ molecule, where the boron atom is close to the oxygen atom. Semiconducting $(8,0) \mathrm{BC}_{2} \mathrm{~N}$ type 2 nanotube that binds to an $\mathrm{H}_{2} \mathrm{O}$ molecule, where (D1) $\mathrm{BN}_{2}$ group binds to an $\mathrm{H}_{2} \mathrm{O}$ molecule with the boron atom close to the oxygen atom, and (D2) $\mathrm{B}_{2} \mathrm{~N}$ group binds to an $\mathrm{H}_{2} \mathrm{O}$ molecule with the nitrogen atom close to the oxygen atom [43]. 
TABle 3: Calculated data for adsorption of $\mathrm{H}_{2} \mathrm{O}$ on the doped carbon nanotubes or $\mathrm{B}_{x} \mathrm{C}_{y} \mathrm{~N}_{z}$ Nanotubes [43].

\begin{tabular}{|c|c|c|c|c|c|}
\hline & $E_{g}(\mathrm{eV})^{\mathrm{f}}$ & Configuration & $E_{b}(\mathrm{eV})$ & $D(\AA)$ & $E T(\mathrm{el})^{\mathrm{g}}$ \\
\hline $\mathrm{CNT}$ & 0.56 & $\mathrm{H}_{2} \mathrm{O}$ & \multicolumn{3}{|c|}{ no binding } \\
\hline $\mathrm{B}-\mathrm{CNT}^{\mathrm{a}}$ & 0.44 & $\mathrm{~B}-\mathrm{H}_{2} \mathrm{O}^{\mathrm{h}}$ & -0.56 & 1.70 & -0.12 \\
\hline $\mathrm{N}-\mathrm{CNT}^{\mathrm{b}}$ & 0.43 & $\mathrm{~N}-\mathrm{H}_{2} \mathrm{O}^{\mathrm{i}}$ & -0.23 & 3.12 & -0.02 \\
\hline \multirow[t]{2}{*}{$\mathrm{BN}-\mathrm{CNT}^{\mathrm{c}}$} & 0.48 & $\mathrm{~B}-\mathrm{H}_{2} \mathrm{O}$ & -0.48 & 1.72 & -0.20 \\
\hline & & $\mathrm{N}-\mathrm{H}_{2} \mathrm{O}$ & -0.24 & 3.14 & -0.02 \\
\hline $\mathrm{B} 2 \mathrm{~N}-\mathrm{CNT}^{\mathrm{d}}$ & 0.44 & $\mathrm{~N}-\mathrm{H}_{2} \mathrm{O}$ & -0.64 & 3.15 & -0.28 \\
\hline $\mathrm{BN} 2-\mathrm{CNT}^{\mathrm{e}}$ & 0.42 & B- $\mathrm{H}_{2} \mathrm{O}$ & -0.53 & 1.51 & -0.08 \\
\hline \multirow[t]{2}{*}{$\mathrm{BC}_{2} \mathrm{~N}$-type 2} & 0.93 & $\mathrm{~B}-\mathrm{H}_{2} \mathrm{O}$ & -0.48 & 1.72 & -0.21 \\
\hline & & $\mathrm{N}-\mathrm{H}_{2} \mathrm{O}$ & -0.18 & 3.14 & -0.05 \\
\hline
\end{tabular}

${ }^{\text {a }}$ Intrinsic nanotube doped with boron atom in 2-unit cell.

${ }^{\mathrm{b}}$ Intrinsic nanotube doped with nitrogen atom in 2-unit cell.

${ }^{\mathrm{c}}$ Intrinsic nanotube doped with boron-nitrogen atom pair in 2-unit cell.

${ }^{\mathrm{d}}$ Intrinsic nanotube doped with boron-nitrogen-boron atom pair in 2-unit cell.

${ }^{\mathrm{e}}$ Intrinsic nanotubes doped with nitrogen-boron-nitrogen atom pair in 2unit cell.

${ }^{\mathrm{f}}$ HOMOLUMO band gap.

EElectron charge transfer from the nanotube to molecules.

${ }^{\mathrm{h}} \mathrm{H}_{2} \mathrm{O}$ molecule binds to the nanotube with the oxygen atom close to the boron atom.

${ }^{i} \mathrm{H}_{2} \mathrm{O}$ molecule binds to the nanotube with the oxygen atom close to the nitrogen atom.

thermopower, resistance, and density of states of single SWNT or SWNT bundles all showed significant change when exposed to certain gases, as discussed in Section 3. Since sensors based on resistance change as output are easy to build, test, and calibrated, resistance sensors are the most commonly used architecture in the design of CNTs gas sensors.

4.1.1. Semiconducting CNTs FET Gas Sensors. As addressed in a previous section, SWCNTs can be either semiconducting or metallic. If two metal contacts are connected to each end of an individual semiconducting SWNT (SSWCNT), the metal/S-SWCNT/metal device exhibits p-type transistor characteristics. Therefore, the resistance response of CNTs to gas adsorption can be detected with field emit transistors (FETs) [46-50]. Kong et al. [46] built an SSWCNT transistor with a single SWNT for chemical sensing. The device was fabricated by controlled CVD growth of individual SWCNTs on $\mathrm{SiO}_{2} / \mathrm{Si}$ substrates with patterned catalyst islands. Polysilicon in the back side acted as gate of the transistor. Figure 12 shows the conductance versus time curves of the transistor upon exposure to $\mathrm{NO}_{2}$ and $\mathrm{NH}_{3}$ at room temperature. A sharp increase in conductance of the S-SWCNT FET by about three orders of magnitude was observed after introducing $200 \mathrm{ppm}$ of $\mathrm{NO}_{2}$ into the testing chamber (Figure 12(a)). The response time was about 2-10 seconds, and the sensitivity (the ratio between resistance after and before gas exposure) was about 100 to 1000 . When the same sample was exposed to a $1 \% \mathrm{NH}_{3}$ flow after recovery, the conductance of the device decreased dramatically within 1 to 2 minute(s), and the sensitivity was about 10 to 100 (Figure 12(b)).

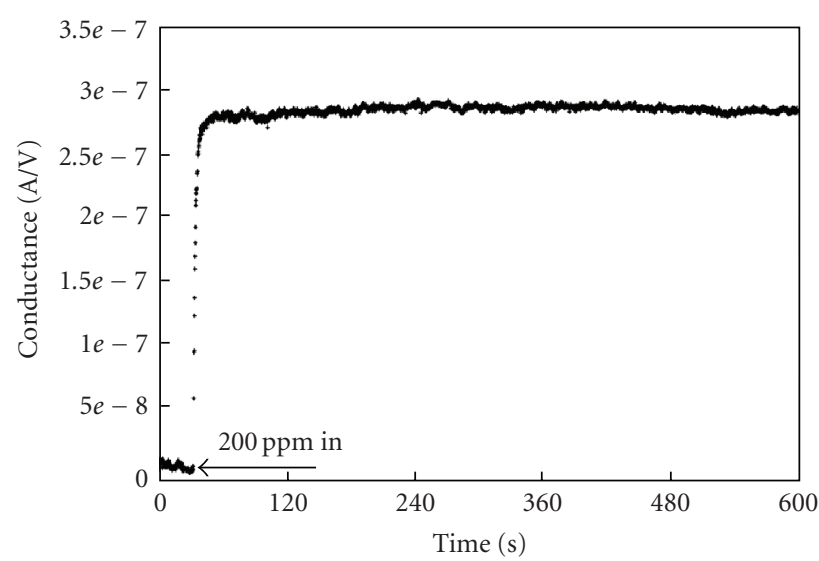

(a)

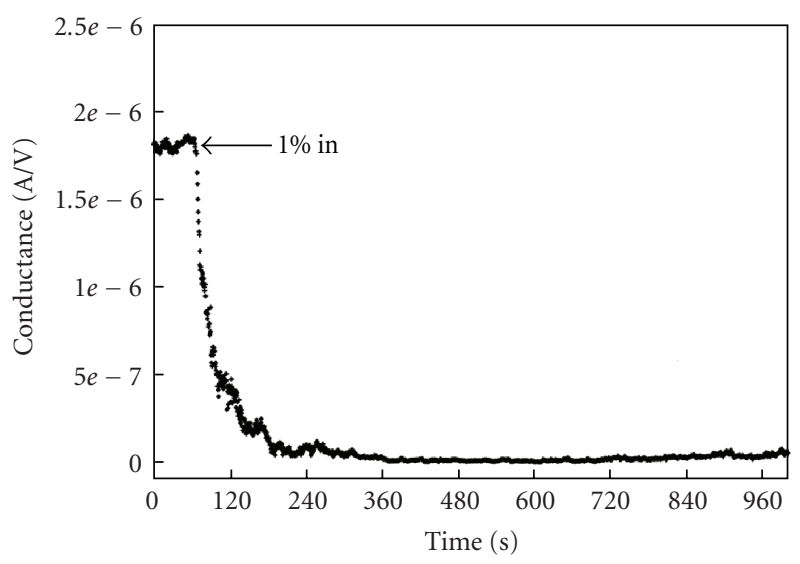

(b)

FIGURE 12: Conductance change of the FET sensor when exposed to (a) $\mathrm{NO}_{2}$ and (b) $\mathrm{NH}_{3}$ gases [46].

Someya et al. [47] characterized the influence of alcohols adsorption on the resistance of SWCNTs using the same transistor structure (see Figure 13). The measurements were carried out under flow of dry nitrogen with and without addition of alcoholic vapour. Figure 14 shows the drain current versus time curves of the device upon exposure to different saturated alcoholic vapour at room temperature (except for tertiary-butanol) with $V g=-20 \mathrm{~V}$. For the vapour of methanol, tertiary-butanol, 1-propanol, 2propanol, and ethanol, the response time was within 5-15 minutes and the current reduction were in the range of $50 \%-90 \%$. Short recovery time (less than few seconds) and excellent repeatability can be obtained if the gate voltage is released briefly and then restored before the next cycle. No degradation of the sensor performance was observed over 1 hour operation. Moreover, the different response characteristic of the device to different alcoholic vapour also suggests its potential application on the identification of gas sources.

Compared with SWCNTs, the mechanism of MWCNTs' response to gas adsorption is more complicated due to the multilayer tube structure. However, they also show high sensitivity to specific gases experimentally $[48,49]$. 


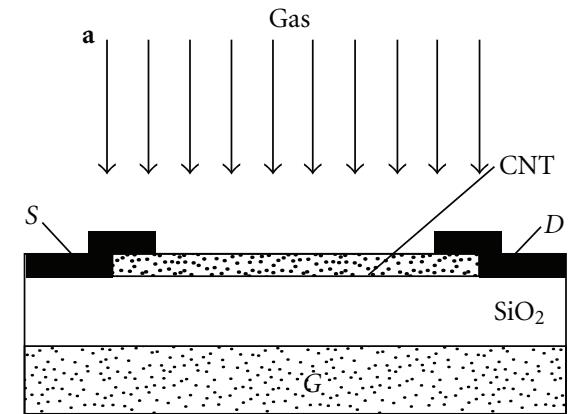

FIgURE 13: Scheme of the SWCNTs based FET sensor [47].

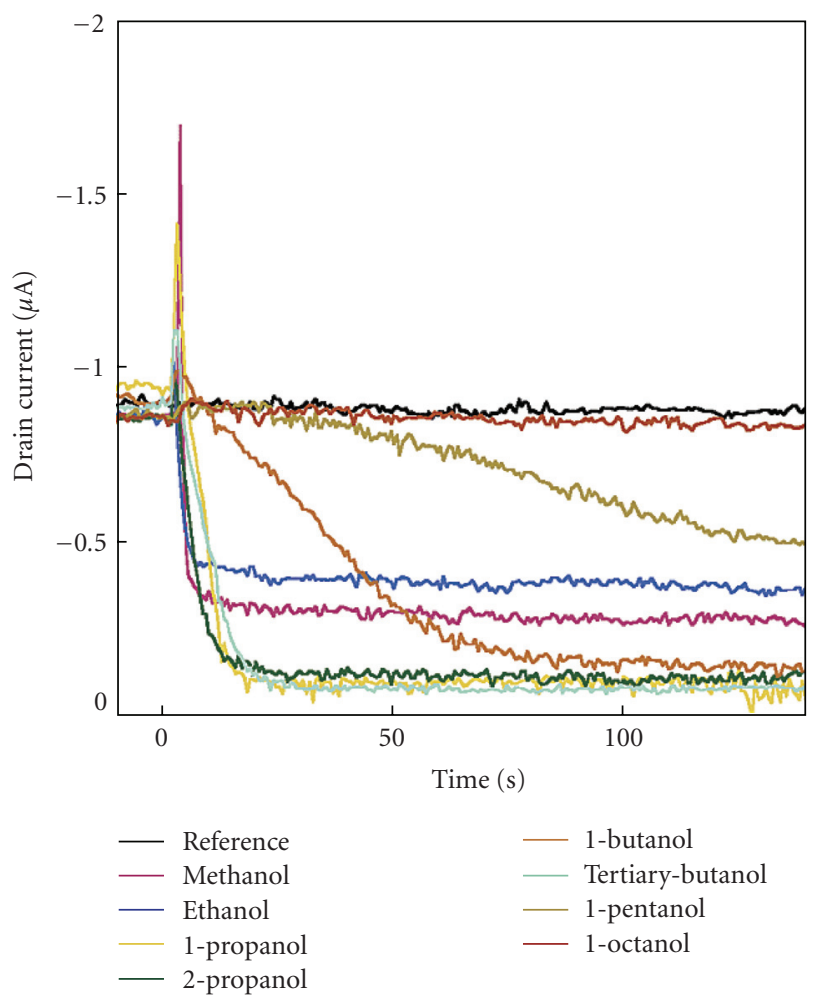

Figure 14: Drain currents a function of time upon exposure to saturated vapour of various kinds of alcohols: methanol, ethanol, 1propanol, 2-propanol, 1-butanol, tertiary-butanol, 1-pentanol, and 1-octanol [47].

Huang et al. [25] synthesized vertically aligned MWNTs mat by thermal CVD and tested their resistance sensitivity to $\mathrm{N}_{2}$ with the FET structure. The MWNTs showed ptype semiconducting property and the source and drain resistance increased when exposed to $\mathrm{N}_{2}$. Figure 15(a) shows the electrical resistance between source and drain at $10 \mathrm{~V}$ bias voltages without any gate voltage under various $\mathrm{N}_{2}$ filling pressure from 50 mTorr to 500 Torr. The increasing resistance was explained by $\mathrm{N}_{2}$ acting as an electron donor to CNTs and decreasing the concentration of conducting holes on CNTs. The sensor showed relative fast response and the resistance returned back to its initial value in vacuum, which indicated $\mathrm{N}_{2}$ molecules were physisorbed

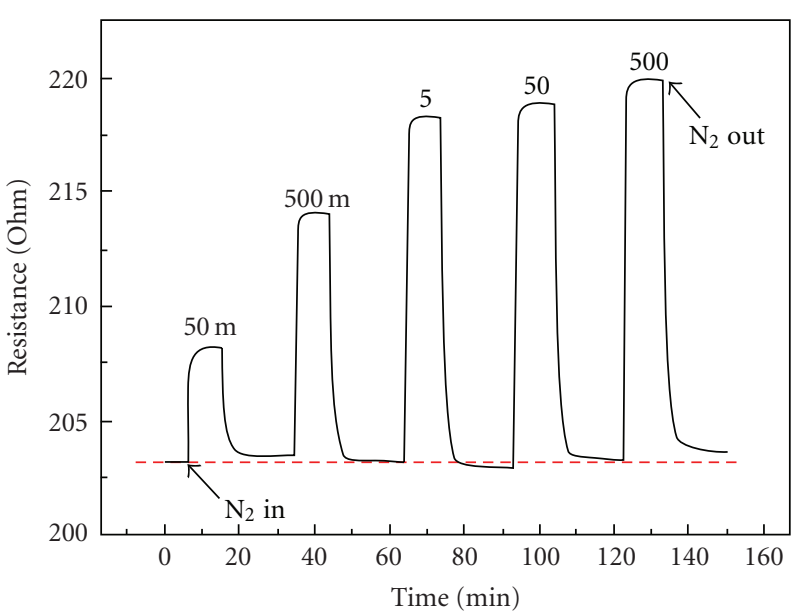

(a)

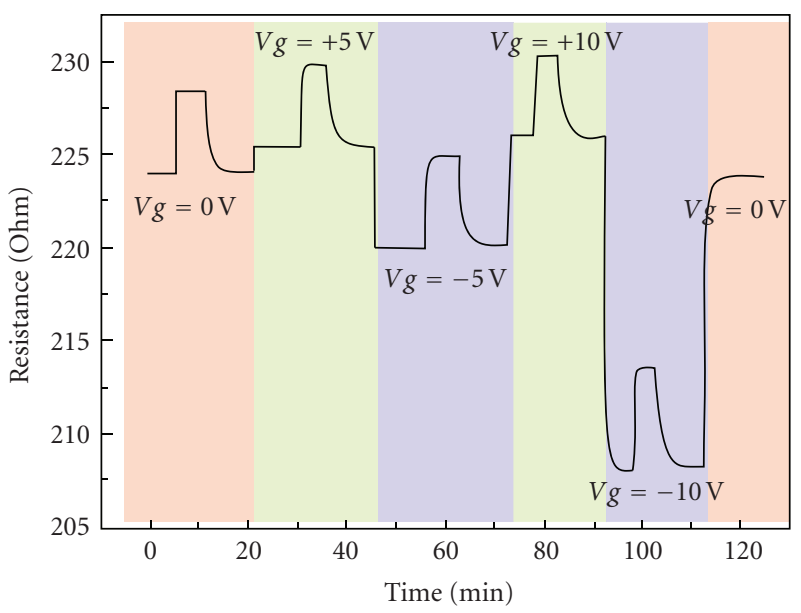

(b)

FIGURE 15: (a) The electrical resistance between source and drain at $10 \mathrm{~V}$ bias voltage without any gate voltage under various $\mathrm{N}_{2}$ filling pressure from $50 \mathrm{~m}$ Torr to 500 Torr. (b) The electrical resistance of CNTs mat measured at a fixed $5 \mathrm{~V}$ source drain bias voltage while applying various gate voltages under a 5 Torr $\mathrm{N}_{2}$ pumping and filling environment [25].

to the CNTs mat. The effect of the gate voltage on the sensor's behaviour was also studied. Figure 15(b) shows the electrical resistance of CNTs mat measured at a fixed $5 \mathrm{~V}$ source drain voltage and various gate voltages, under a 5 Torr $\mathrm{N}_{2}$ pumping and filling environment. The initial electrical resistance increased when the positive gate voltage was applied and decreased with negative voltage. The reason was that electrons (holes) induced by positive (negative) gate voltage neutralized (added) some conducting holes in p-type CNTs, which caused the change of the free holes concentration and the electrical resistance of the CNTs mat. The recovery of the resistance to its initial value after a cycle of $\mathrm{N}_{2}$ filling and pumping was observed during different positive and negative gate voltages. It was also demonstrated that the sensor sensitivity increased with the decrease of the gate voltage from positive to negative and the trend was more 


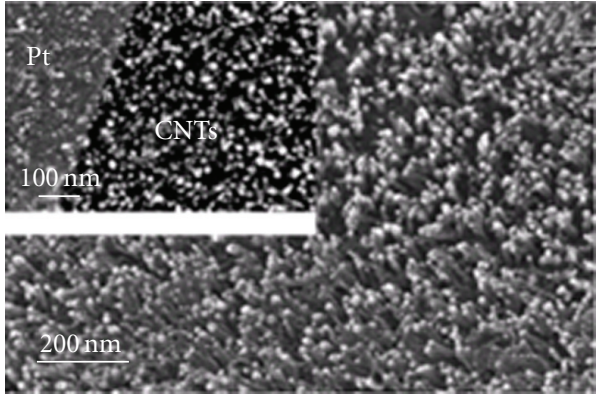

(a)
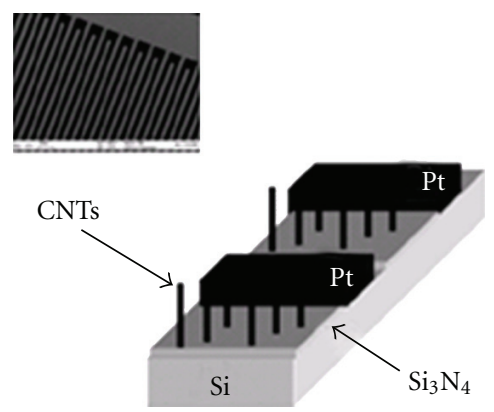

(b)

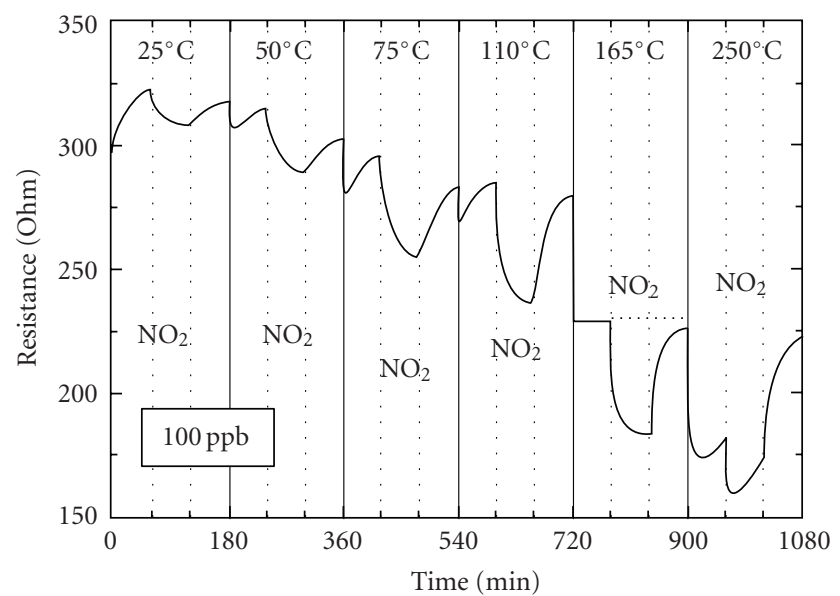

(c)

FIGURE 16: (a) SEM image of the as-grown structure of CNTs on $\mathrm{Si} / \mathrm{Si}_{3} \mathrm{~N}_{4}$; (b) schematic diagram of CNTs linking pre-patterned platinum contacts in resistor geometry; (c) dynamic gas responses of CNTs thin film at different operating temperatures in dry air and $100 \mathrm{ppb}$ of $\mathrm{NO}_{2}$ [52].

obvious under higher $\mathrm{N}_{2}$ pressure. This was believed due to the increase of the gas-binding sites caused by negative gate voltage.

4.1.2. CNTs-Based Two Terminal Resistor Gas Sensors. The resistance change of CNTs under gas exposure can also be detected by two terminal resistors with dc voltage. Interdigitated electrodes are generally applied in these kind of sensors to provide larger sensing areas and sufficient contacts between the electrodes and the coated CNTs [51]. Valentiniet et al. [52] designed a CNT serpentine resistor for the detection of various gases including $\mathrm{NO}_{2}, \mathrm{CO}, \mathrm{NH}_{3}, \mathrm{H}_{2} \mathrm{O}$, and $\mathrm{C}_{2} \mathrm{H}_{5} \mathrm{OH}$. The sensor was fabricated by photolithography defining Pt IDEs on $\mathrm{Si}_{3} \mathrm{~N}_{4}$ substrate, and then CNTs films were grown on the substrate using radiofrequency plasma enhanced CVD (RF PECVD) with Ni as the catalyst. Figures 16(a) and 16(b)show the as-grown CNTs and a scheme of the sensor design. Figure 16(c) shows the dynamic response of the film resistance at different operating temperature from 25 to $250^{\circ} \mathrm{C}$ and different $\mathrm{NO}_{2}$ concentration with dry air mixture. The sensor showed good sensitivity to $\mathrm{NO}_{2}$ at room temperature. At $165^{\circ} \mathrm{C}$, the sensor showed the best response as a tradeoff between the higher resistance variations and the fast and reproducible baseline recovery. The sensor's resistance response of other gases was also studied. In summary, $\mathrm{NO}_{2}$ exposure drastically decreases the electrical resistance; $\mathrm{NH}_{3}, \mathrm{H}_{2} \mathrm{O}, \mathrm{C}_{6} \mathrm{H}_{6}$ and ethanol exposure increases the electrical resistance; $\mathrm{CO}$ exposure does not affect the resistance; removing the gas exposure totally restores the initial resistance.

Li et al. developed a similar gas sensor by simply casting SWCNTs on IDEs [14]. The sensor is shown in Figure 17. Upon exposure to $\mathrm{NO}_{2}$, the conductance change of the sensor showed a linear response to the gas concentration with a detection limit of less than $4 \mathrm{ppb}$. The recovery time was about 10 hours but can be intensively shortened by UV light treatment, as shown in Figure 17. The sensor's response to organic vapour such as benzene, acetone, and nitrotoluene was also measured.

Wong et al. [53] built a microelectronic gas sensor with CNTs in a thin-layered $\mathrm{Pd} / \mathrm{CNTs} / \mathrm{n}^{+}-\mathrm{Si}$ structure for hydrogen detection. The sensor was fabricated on an n-type silicon wafer, which acted as an ohmic supporting substrate. MWCNTs were grown selectively on the substrate via catalytic activation with microwave PECVD. Annealing of the CNTs layer was carried out to improve the adhesion with the silicon substrate. A thin layer of Pd was then sputtered on top of the CNTs layer as the gate electrode. The $I-V$ characteristic of the device exhibit Schottky diode behaviour at room temperature with instantaneous current changes (reduction) when it was exposed to constant flow of hydrogen gas at room temperature, as shown in Figure 18(a). The reduction of the current in the presence of hydrogen was believed to be due to an increase in the barrier height between the PdCNTs interface after $\mathrm{H}_{2}$ adsorption. The sensor was also detected at elevated temperature. Increasing current change was observed with increasing operating temperature. The sensor still showed Schottky diode behaviour at $80^{\circ} \mathrm{C}$, while it changes to ohmic in both open air and $\mathrm{H}_{2}$ environment at $170^{\circ} \mathrm{C}$, as shown in Figure $18(\mathrm{~b})$. This was explained by the change in the electrical properties of the CNTs at elevated temperature.

4.2. Carbon Nanotubes Enhanced Ionization Chamber for Gas Sensing. In the case of chemical gas sensors, it is difficult to detect gas molecules with low adsorption energy. Compared to these sensors, ionization gas sensor is based on the fingerprinting ionization characteristics of the detected gases. The ionization of detected gas is caused by the collisions of molecules with accelerated electrons. There is no adsorption 


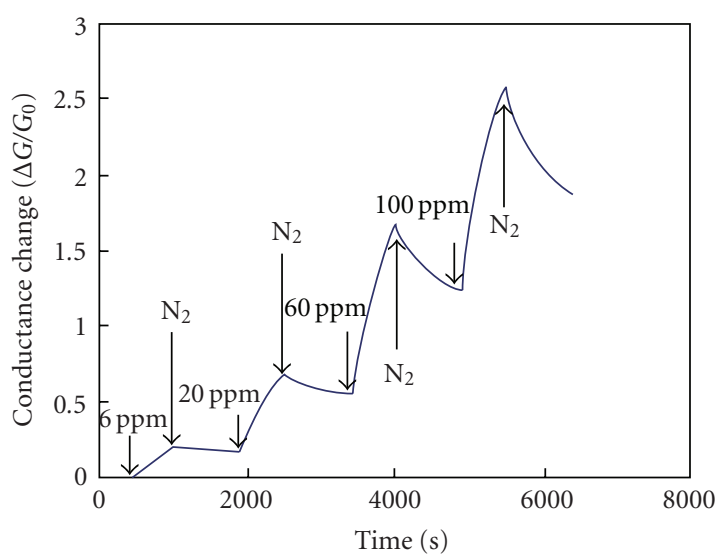

(a)

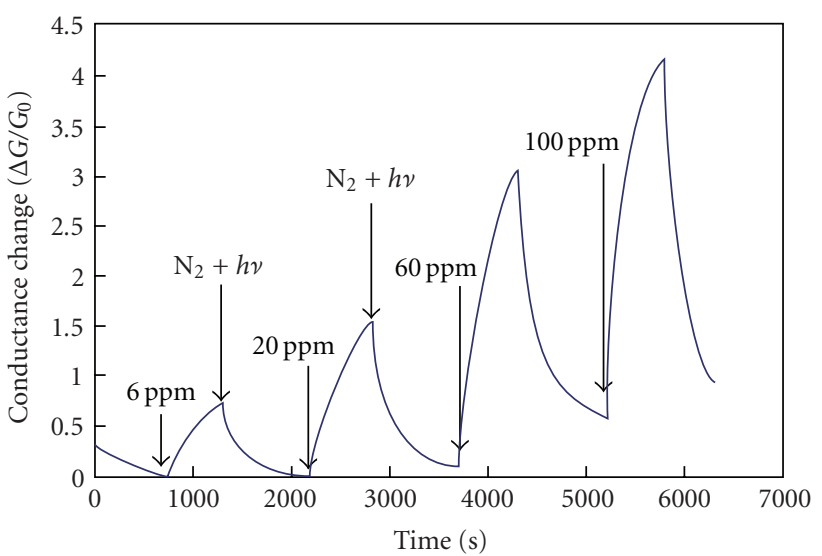

(b)

FIgURE 17: Representative sensor response $\mathrm{NO}_{2}$. Various concentrations of the sample gas and the pure gas injections are shown by arrows. The sample gas is $\mathrm{NO}_{2}$ in a $400 \mathrm{~cm}^{3} / \mathrm{min}$ nitrogen flow at room temperature. Ultrapure nitrogen is used for dilution and purging. (a) Without UV light in the recovery. (b) With UV light for sensor recovery. The UV illumination and $\mathrm{N}_{2}$ purging start simultaneously [14].

and chemical interaction between the device and interested molecules. Therefore, they are not limited to identify gases with low adsorption energy and poor charge transfer with the sensing materials. Sensing of inert gases or gas mixtures can easily be achieved by ionization chamber. However, the issues related to conventional ionization gas sensors are their bulky architectures, considerable high power consumption, and breakdown voltage, which is inefficient and risky in operation. CNTs, due to their extremely sharp tip structure, can induce a large field enhancement factor and thereby intensively increase the electric field around the tips to initiate corona discharge at very low voltage [54]. Therefore, the effect of gas adsorption on their field emission properties and CNTs enhanced ionization gas sensors have attracted a great amount of research interests [55-62].

A miniaturized gas ionization sensor with aligned MWNT film as anode was developed by Ashish Modi et al. in 2003 [55]. Figure 19 shows a diagram of the sensor

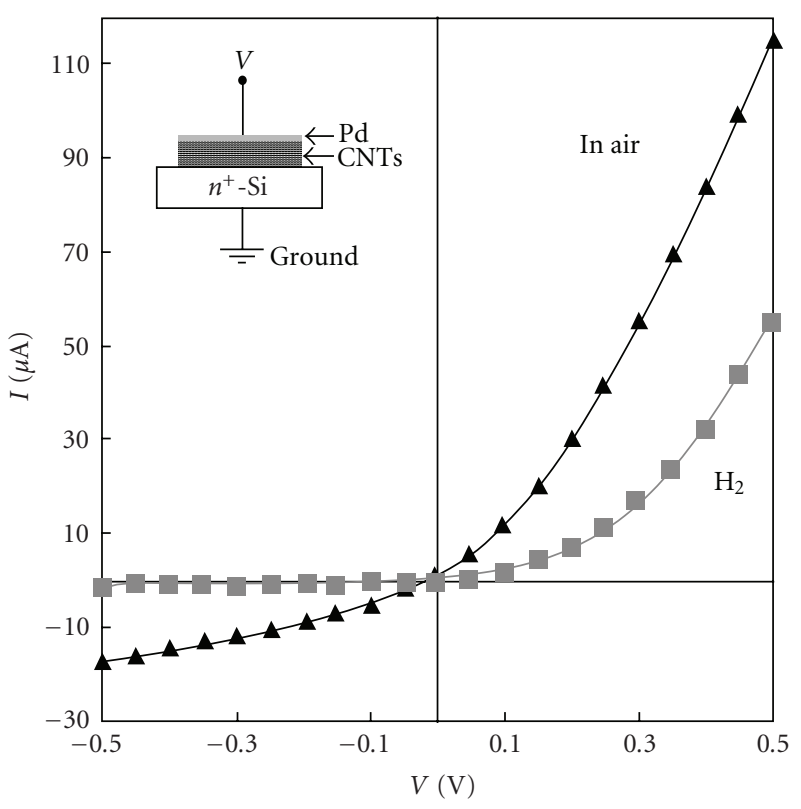

(a)

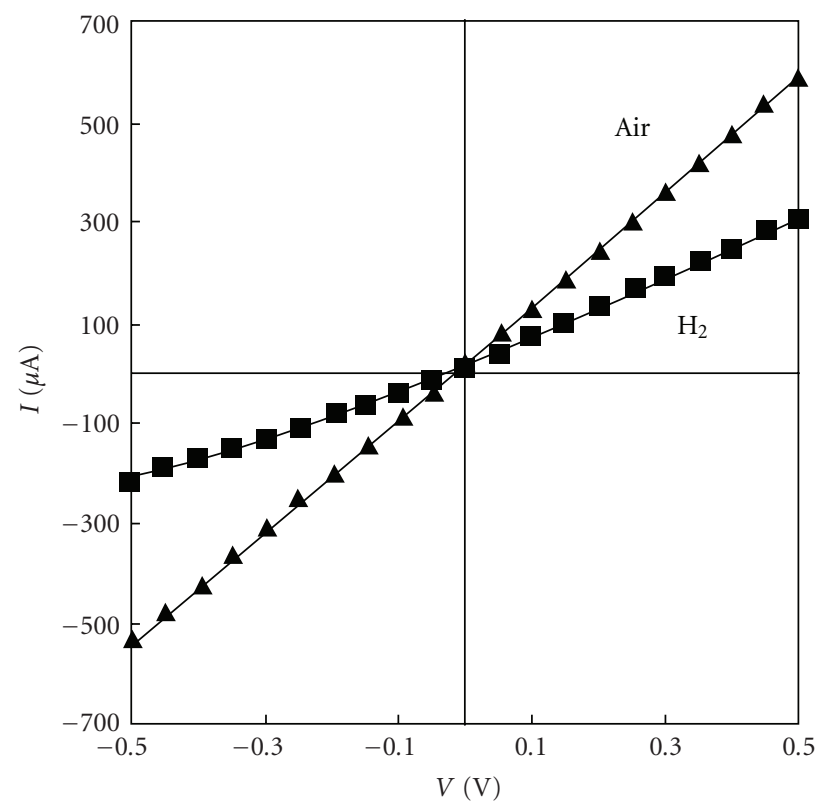

(b)

Figure 18: (a) Plot of measured current versus applied voltage ( $I$ $V)$ of the CNTs-based sensor in air and after exposure to constant flow of $\mathrm{H}_{2}$ (concentration, $100 \%$; flow rate, $10 \mathrm{~mL} / \mathrm{min}$ ) at room temperature. (b) Plot of measured current versus applied voltage ( $I$ $V$ ) of the CNTs-based sensor in air and after exposure to constant flow of $\mathrm{H}_{2}$ (concentration, $100 \%$; flow rate, $10 \mathrm{~mL} / \mathrm{min}$ ) at $170^{\circ} \mathrm{C}$ [53].

structure. It consists of MWNT film anode, Al plate cathode and a $150 \mu \mathrm{m}$ thick glass insulator between them. The vertically aligned MWNT film was prepared by CVD on $\mathrm{SiO}_{2}$ substrate with tip diameter in the range of $25 \sim 30 \mathrm{~nm}$ and $30 \mu \mathrm{m}$ in length (Figure $19(\mathrm{c})$ ). During operation, individual MWNTs create very high nonlinear electric fields 


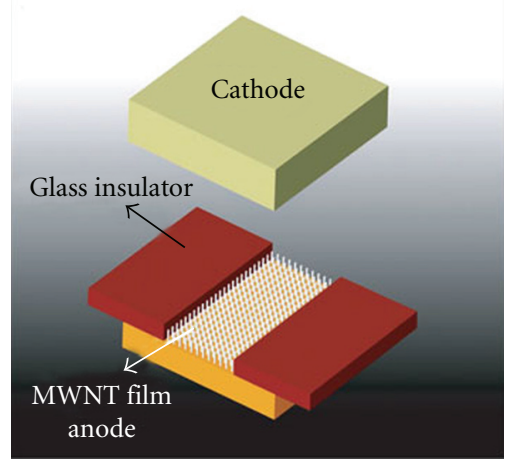

(a)

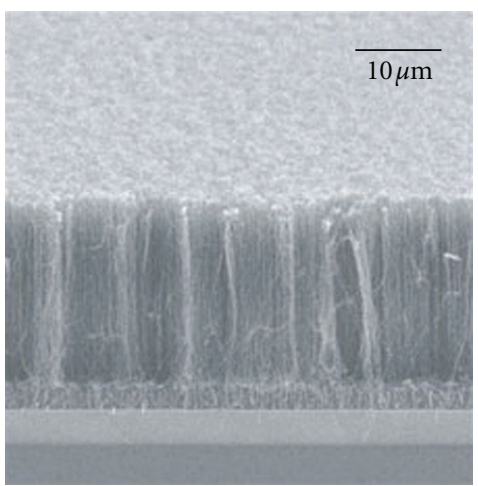

(c)

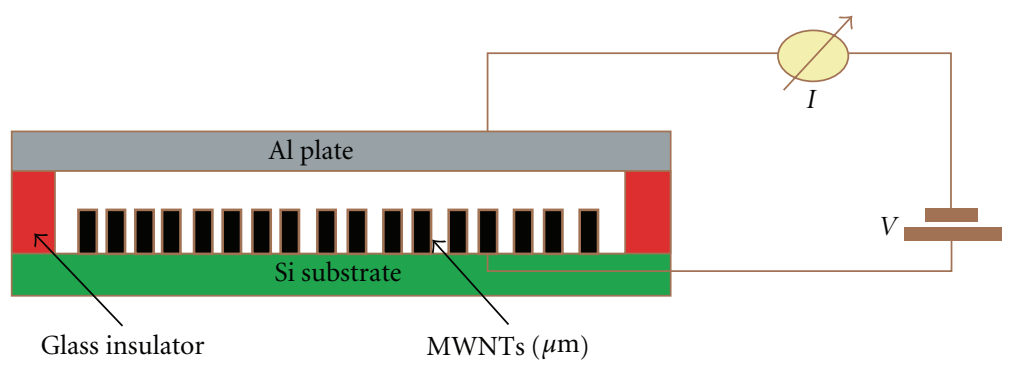

(b)

FIGURE 19: (a) Schematic diagram of the nanotube sensor device; (b) diagram of actual test set-up; (c) SEM micrograph of vertically aligned MWCNT film used as the anode [55].

near the tips $[56,57]$. This results in the formation of a "corona" or conduction filament of high ionized gas that surrounds the MWNT tips, and thereby promotes the formation of a self-sustaining interelectrode discharge at very low voltage. Compared with ionization chamber with metal anode (without CNTs), the breakdown voltage of air was brought down dramatically from $960 \mathrm{~V}$ to $346 \mathrm{~V}$. Different gases including $\mathrm{NH}_{3}, \mathrm{CO}_{2}, \mathrm{~N}_{2}, \mathrm{O}_{2}, \mathrm{He}, \mathrm{Ar}$, and air were tested and showed distinct and precise breakdown voltage (Figure 20).

The precise breakdown voltage provides a "fingerprint" to each gas and indicates the potential for gas identification within a mixture of gases. The discharge current of each gas showed almost linear relationship with the gas concentration, but the breakdown voltage was rarely affected by the concentration (see Figure 21). This was explained by the fact that the discharge current is dominated by the number of molecules per unit volume and the breakdown voltage depends mainly on the intensity of electric field and the bonding energy of the gas molecules.

Nanotubes are good electron emitter due to their sharp tip curvature and low electron escaping work function. If CNT films are deposited as cathode of the device instead of anode, the breakdown process will be initiated by a negative corona. Field emission may occur under low pressure to enhance the ionization, and lower breakdown voltage can be obtained as a result. Kim [58,59] designed an ionization chamber with CNTs as the emitters for the detection of gases with low adsorption energy. The fabrication process and structure of the sensor are shown in Figure 22. CNTs were

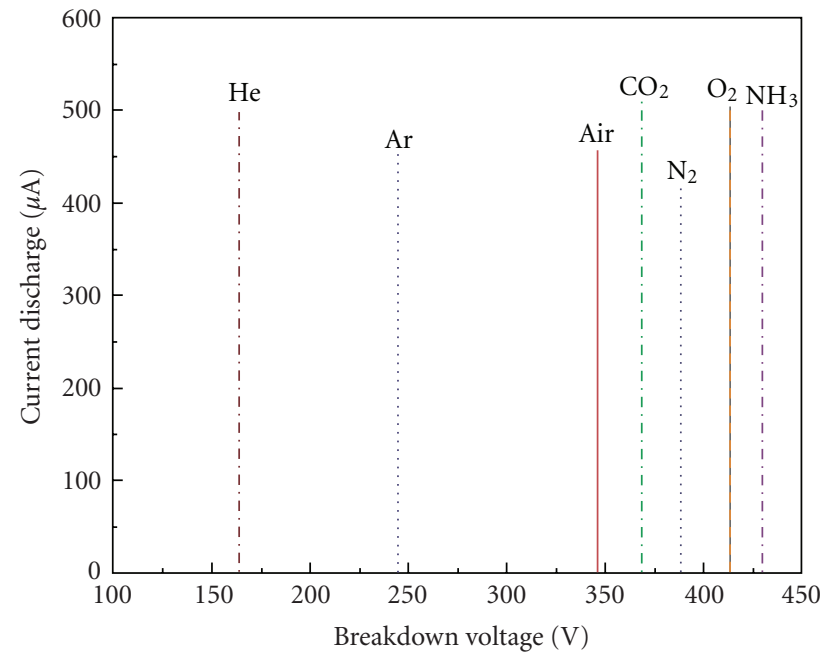

Figure 20: Current-voltage curves for electrical breakdown for $\mathrm{NH}_{3}, \mathrm{CO}_{2}, \mathrm{~N}_{2}, \mathrm{O}_{2}, \mathrm{He}, \mathrm{Ar}$ and air [55].

grown on n-type silicon substrate by CVD and served as the cathode. During operation, once electrons are emitted from the CNTs under certain voltage, they are accelerated towards the anode, collide with gas molecules, and induce ionization of the neural molecules. Eventually it will lead to a current multiplication of breakdown. By Paschen's law, the initial sparking breakdown voltage in an air gap is a function of the product of gas pressure and the electrodes distance. 


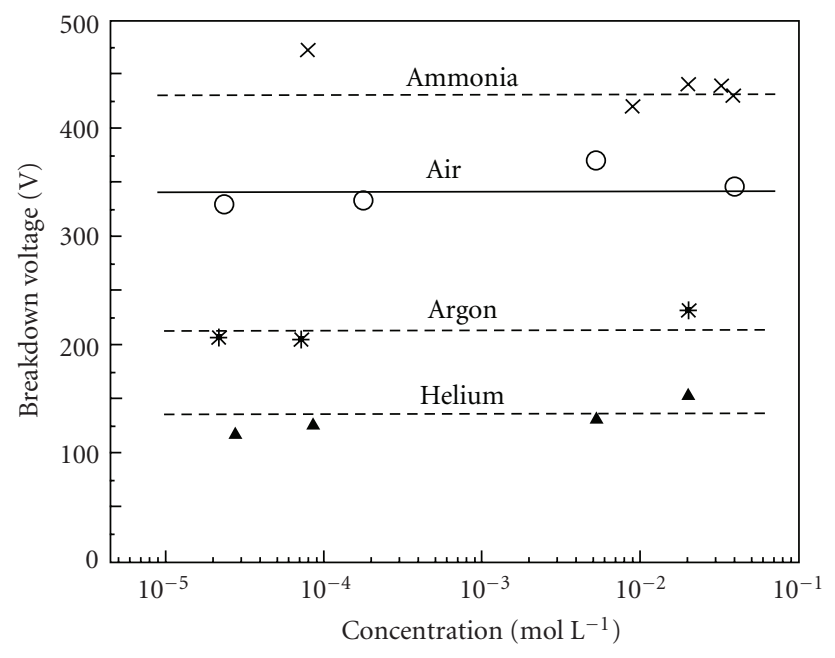

(a)

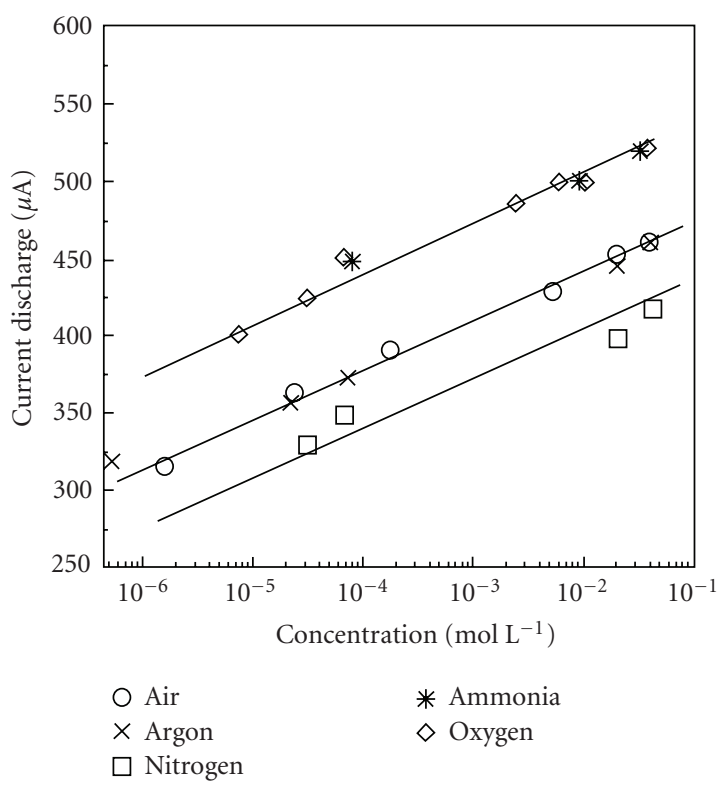

(b)

FIGURE 21: Effect of gas concentration on the electrical breakdown. (a) Breakdown voltage as a function of concentration; breakdown voltages vary only slightly with gas concentration; (b) discharge current at breakdown as a function of gas concentration. The discharge current varies logarithmically with concentration [55].

Therefore, the initial breakdown voltages are measured as a function of the gas concentration. Figure 23 shows the dependence of the initial breakdown voltage on air concentration in the chamber. On contrary with Madi's results [55], the breakdown voltage decreases with increasing concentration and then increase again. The decreasing of breakdown voltage at first was explained by the dependency of the discharge current on the amount of neutral molecules, which can provide electron-ion pairs generated by collisions. When the concentration increases to a certain value, the ionization rate is predominantly determined by the length of the mean free path rather than the numbers of the molecules.

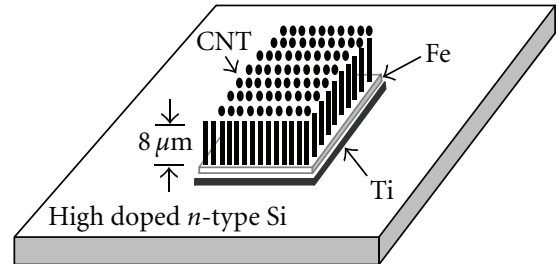

(a)
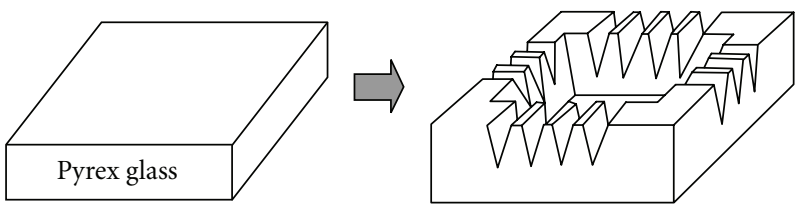

(b)

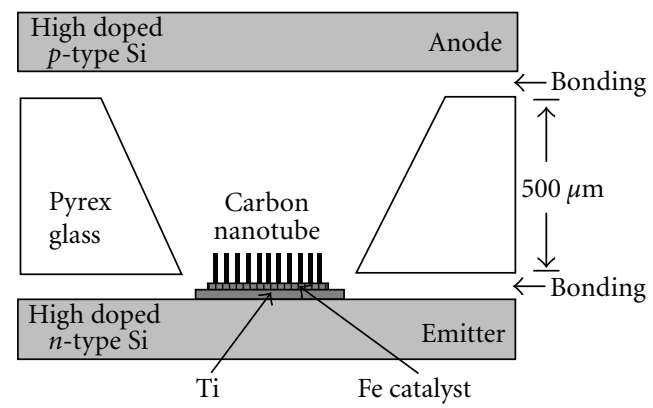

(c)

FIGURE 22: Fabrication process: (a) CNT electron emitters, (b) glass patterning by sand blast method, and (c) Si/glass anodic bonding [58].

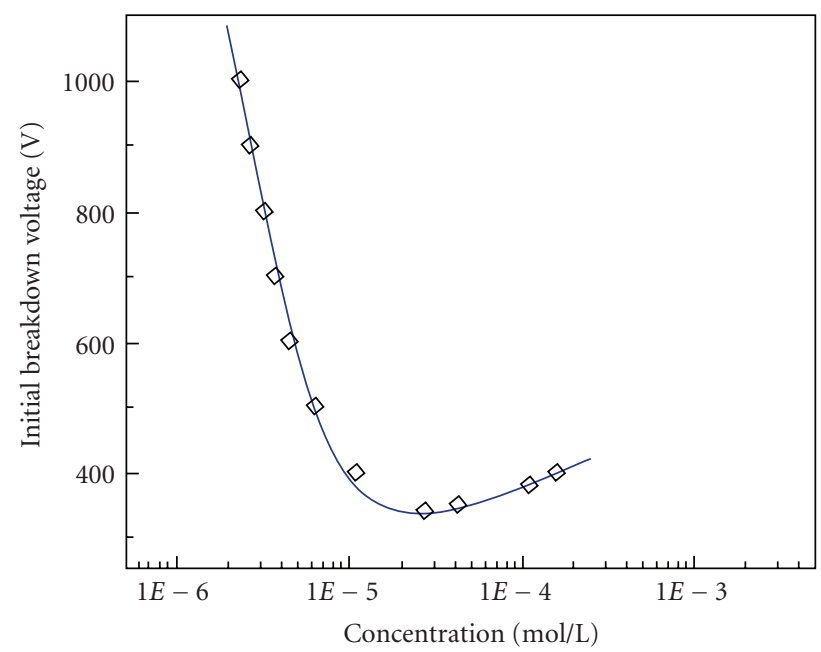

FIGURE 23: Dependence of initial breakdown voltage on air concentration [59].

With higher concentration, which means shorter mean free path, the ionization rate is reduced which results in higher breakdown value [59].

Another important factor that dominates the ionization characteristic is the distance between anode and cathode. According to Paschen's curve, a certain gap distance is 


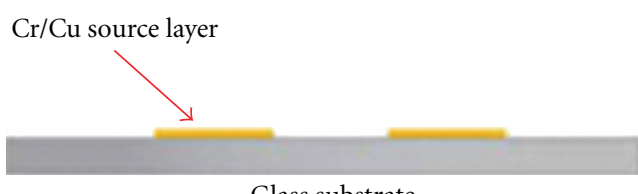

Glass substrate

(a)

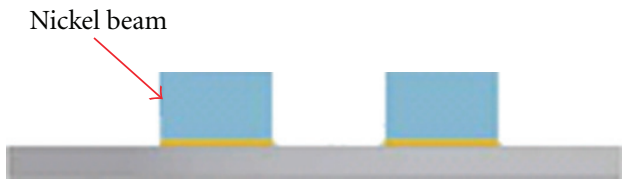

(b)

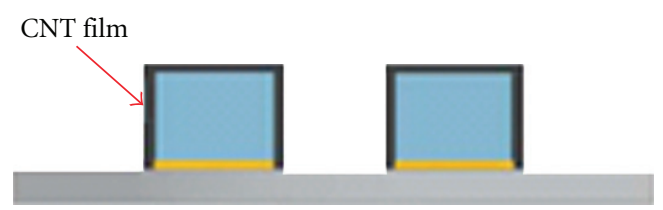

(c)

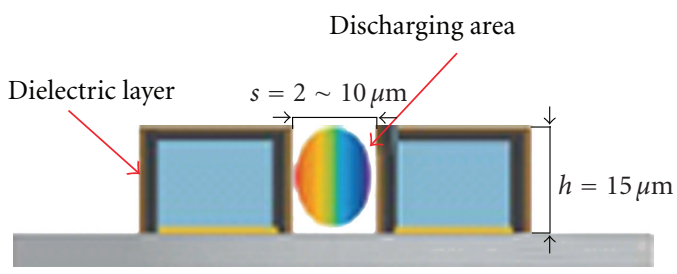

(d)

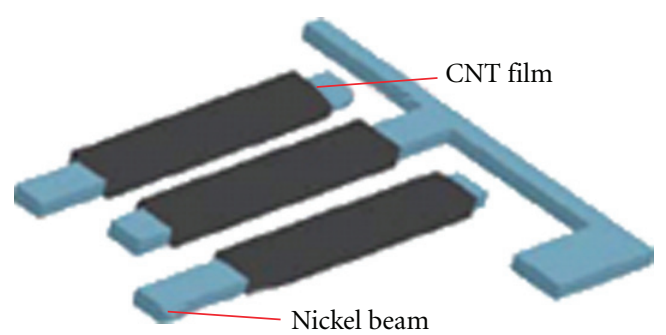

(e)

FIgURE 24: Fabrication process flow and schematic diagram of the sensor [60].

required for breakdown phenomenon to be induced, and the breakdown voltage decreases by bringing the electrodes closer. In the capacitor-architecture device, it is very difficult to control the electrode gap under $10 \mu \mathrm{m}$ to gain a safe operation voltage. Hou et al. [54] introduced a novel hollow slot electrode system with CNT sidewalls and electrode spacing of $6-12 \mu \mathrm{m}$ for gas ionization sensors, which dramatically decreased the threshold voltage to $5 \mathrm{~V}-40 \mathrm{~V}$.

Based on same idea and integrated dielectric barrier discharge (DBD) mechanism, Wu et al. [60] designed and fabricated a novel ionization gas sensor with shortgas spacing, CNTs and DBD coating to realize low power consumption and breakdown voltage. As shown in Figure 24, the IDE electrodes are deposited and patterned on glass substrate. CNTs are deposited by electrophoresis method and

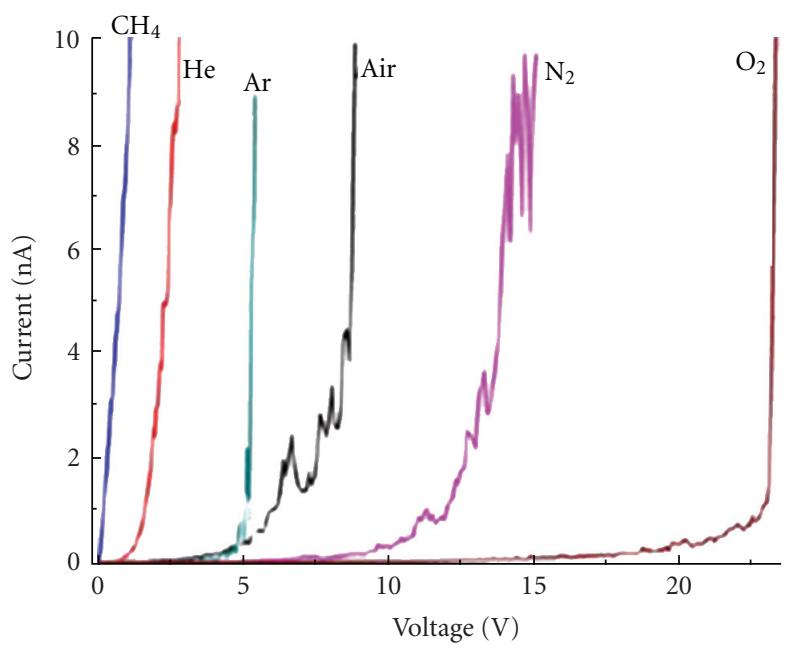

(a)

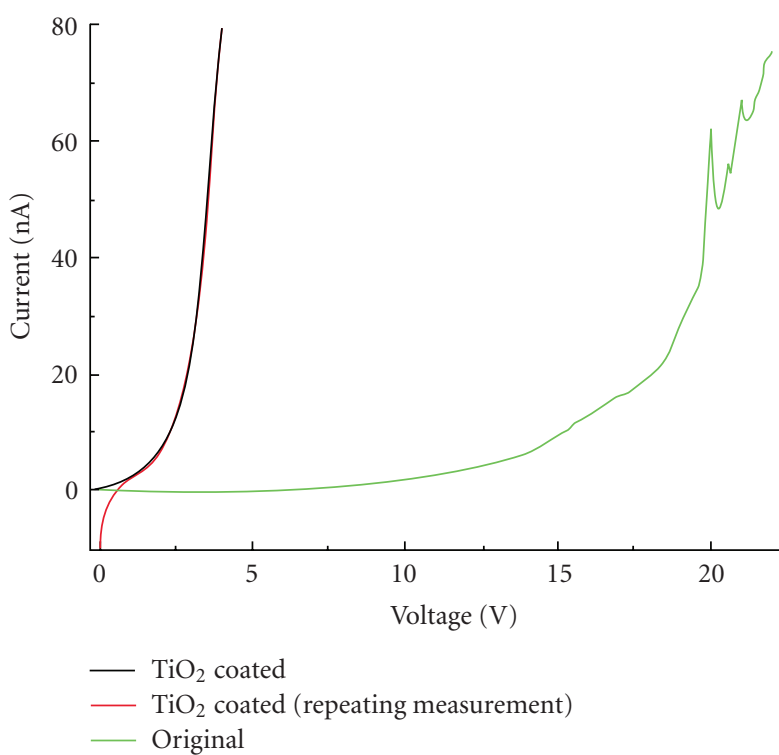

(b)

Figure 25: (a) The discharge $I$ - $V$ curves of 6 different gases. (b) The gas discharge $I-V$ curves of the same device before and after $\mathrm{TiO}_{2}$ coated [60].

then a thin film of $\mathrm{TiO}_{2}$ dielectric barrier was deposited by sputtering on top of CNTs serving as the DBD layer. The results show that the sensor can successfully distinguish different gases by the fingerprinting breakdown voltage, as shown in Figure 25(a). With the device of $8 \mu \mathrm{m}$ spacing gap, the breakdown voltage for air is brought down dramatically to $5 \mathrm{~V}$. It is also demonstrated that the DBD layer effectively lowers the breakdown voltage and improves the reproducibility of the device (Figure 25(b)). The low power consumption and breakdown voltage show a bright future of compact, battery powered, safe operation, and wide range of the applications of CNTs-based ionization gas sensors.

4.3. CNTs Based Gas Sensing Capacitor. Yeow and She [63] demonstrated the use of randomly aligned CNTs-based 

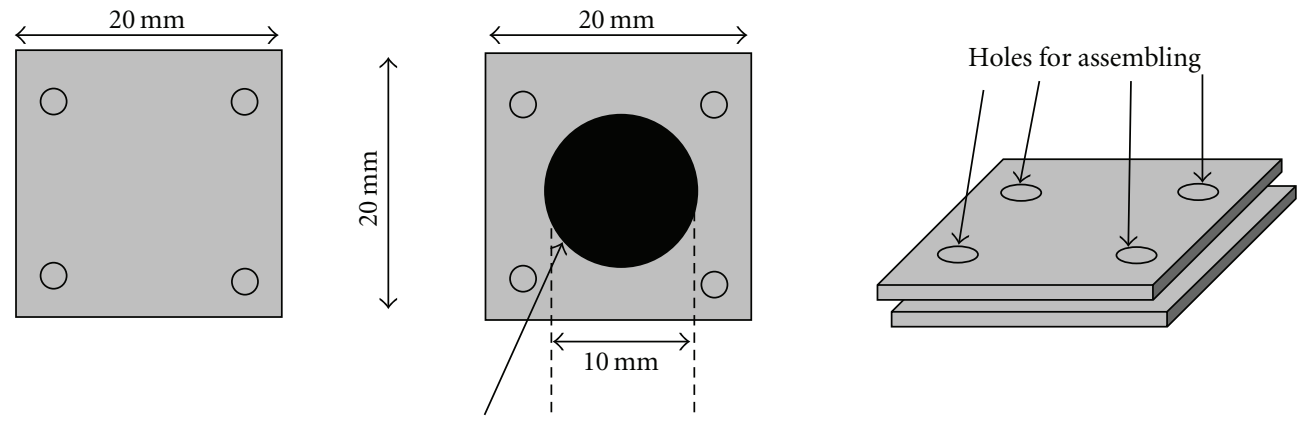

Coated with CNT

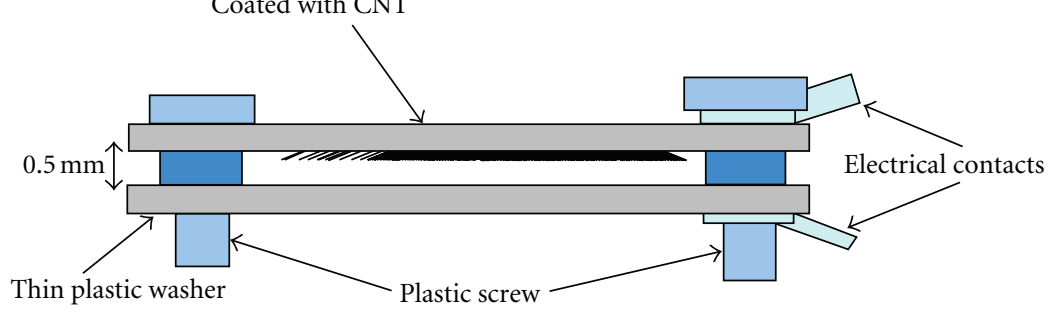

FIgURE 26: Parallel plate capacitive humidity sensor with MWNTs deposited on one plate [63].

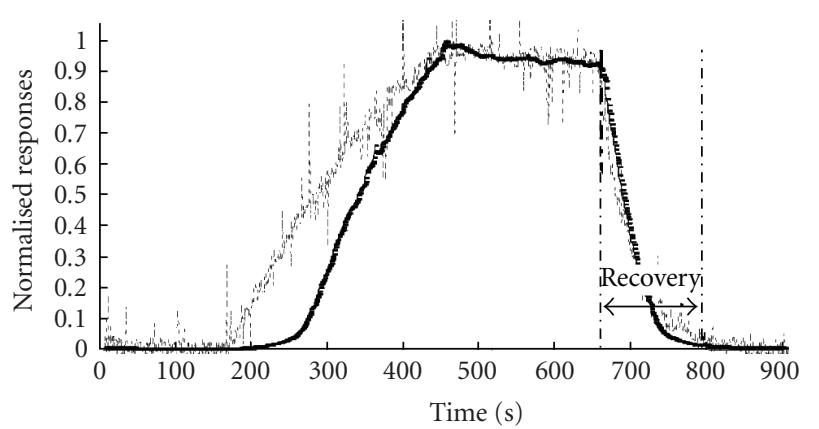

- CNT-enhanced sensor

-..- Honeywell sensor

FIGURE 27: The normalized response of the nanotube and the Honeywell sensors [63].

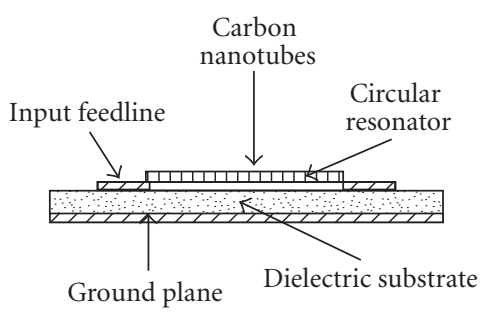

(a)

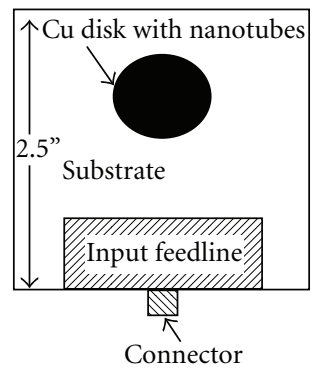

(b)
FIGURe 28: (a) Schematic of front view of the resonator circuit. (b) Top view of the resonator sample coated with nanotubes [65].

capacitor for humidity sensing. The structure of the parallel plate capacitor is shown in Figure 26. The sensor showed linear response to humidity change from $65 \%$ to $80 \%$ and

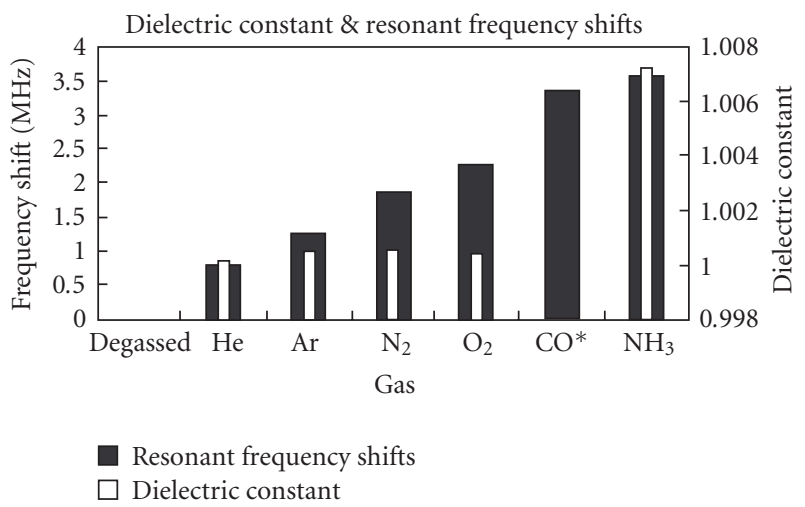

FIGURE 29: Resonant frequency shifts (solid bars) and dielectric constants (open bars) of various polar and nonpolar gases. At this point, room temperature value for dielectric constant of CO is not available in the literature [65].

very fast response and recovery in this sensing range (as shown in Figure 27). It is believed that the increase of the capacitance under high relative humidity is cause by capillary condensation, which is induced by the nanopores and interstitial gaps in the random aligned CNTs structure.

Snow et al. [64] also developed a capacitor with SWNTs and highly dope Si substrate as the two plates. The sensor showed high sensitivity to a wide range of vapours. When excitation voltage is applied between the two plates, a large concentration of electric field is created at the tip of the nanotubes. This results in the polarization of the adsorbed molecules and the increase of the capacitance value.

4.4. Resonance Frequency Shifts. The change of the electrical property of CNTs upon exposure to gases can also be applied to resonator sensor. Chopra et al. $[65,66]$ fabricated a 


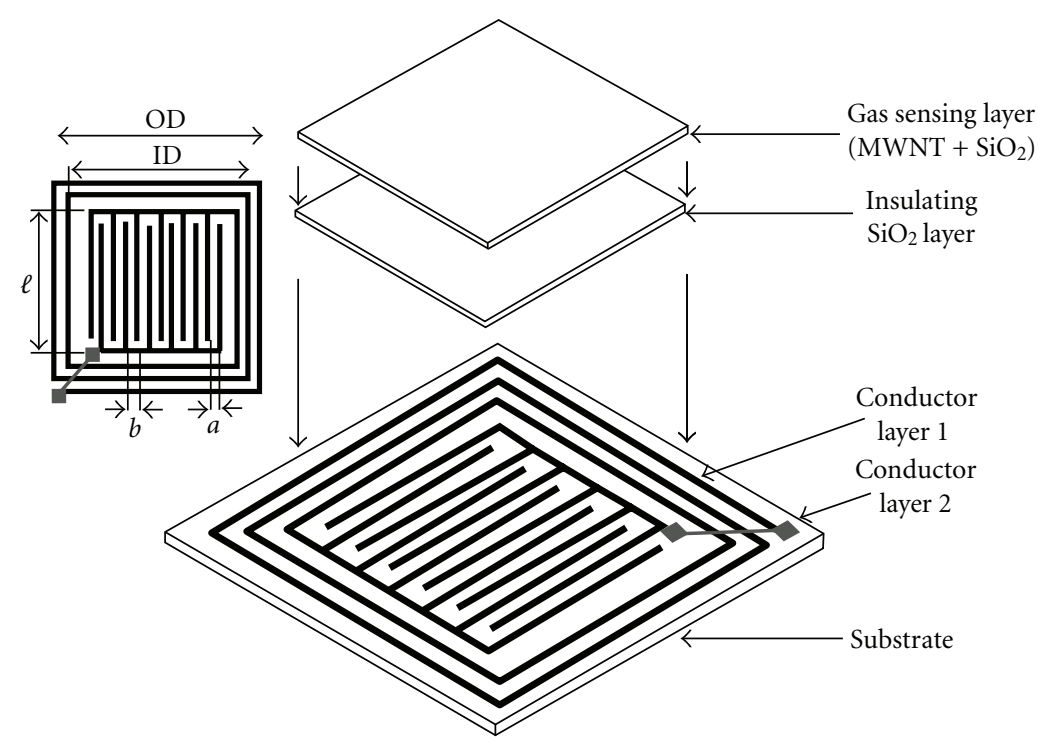

FIGURE 30: Schematic drawing of the MWNT gas sensor. A planar inductor interdigital capacitor pair is photolithographically defined upon a copper clad printed circuit board. The capacitor is first coated with a protective electrically insulating $\mathrm{SiO}_{2}$ layer followed by a layer of gas responsive MWNT-SiO ${ }_{2}$ composite [68].

circular disk resonator with SWNTs coated on top of the disk. The design of the sensor is shown in Figure 28. The input feedline also serves as the output port to an analyzer. According to

$$
f_{0}=\frac{1.841 \mathrm{c}}{2 \pi a \sqrt{\varepsilon_{r}}},
$$

where $f_{0}$ is the resonant frequency of the resonator, $c$ is the speed of light in vacuum, $a$ is the radius of the disk, and $\varepsilon_{r}$ is the relative dielectric constant of the substrate, when the CNTs-based resonator is exposed to certain vapours, the change of the dielectric constant of the circular disk with CNTs on top results in the resonate frequency shift. Figure 29 shows the sensor's response to different gases. The sensor can detect a wide range of vapours including $\mathrm{NH}_{3}, \mathrm{CO}, \mathrm{N}_{2}$, $\mathrm{He}, \mathrm{O}_{2}$, and Ar with low concertrations. Since different gas caused different shift value of the resonate frequency, the sensor poccess a good sensing selectivity.

CNTs-coated surface acoustic waves (SAWs) sensor also showed high sensitivity to volatile organic vapours [67]. Ong et al. [68] built a wireless, inductor-capacitor resonantcircuit (LC) gas sensor with $\mathrm{MWNT}-\mathrm{SiO}_{2}$ composite as the sensing layer. Figure 30 showed the sensor's structure. It consists of a printed LC resonant circuit that is first coated with a protective, electrically insulating $\mathrm{SiO}_{2}$ layer, followed by a second layer of gas-responsive $\mathrm{MWNT}-\mathrm{SiO}_{2}$ mixture with the $\mathrm{SiO}_{2}$ matrix acting to physically bind the MWNTs to the sensor. When the sensor is exposed to various gases, the relative permittivity $\varepsilon_{r}^{\prime}$ and the conductivity (proportional to $\left.\varepsilon_{r}^{\prime \prime}\right)$ of the MWNTs change, resulting in the change of the effective complex permittivity of the coating and hence the resonant frequency of the sensor. The frequency spectrum of the sensor is obtained by a sensor-monitoring loop antenna. It is found that the $\varepsilon_{r}^{\prime \prime}$ of the nanotubes shifted lower when exposed to $\mathrm{CO}_{2}, \mathrm{NH}_{3}$ (which are reducing gases) and higher when exposed to $\mathrm{O}_{2}$ (which is oxidizing agent). This can be explained by the p-type nature of the MWNTs. The change of the nanotubes' permittivity $\varepsilon_{r}^{\prime}$ upon exposure to the gases was caused by the permittivity difference between the agents and the MWNTs. With this wireless, passive, remote query sensor platform, long term monitoring from within sealed containers and environment can be achieved.

\section{Functionalized CNTs and CNTs-Based Composites for Gas Sensing}

5.1. Functionalization of CNTs for Gas Sensing. Covalent sidewall functionalization opens new doors for the application of CNTs in many areas [69-71]. As discussed in Section 2, the electronic sensitivity of CNTs to various gases is highly limited by the binding energy and charge transfers of the molecules with the CNTs wall. It was proved theoretically that by doping CNTs with B-and/or N- and $\mathrm{B}_{x} \mathrm{C}_{y} \mathrm{~N}_{z}$ groups, their electronic properties can be very sensitive to a wide range of gas vapours [43].

Sin et al. [72] demonstrated the resistance response of chemically functionalized MWNTS (f-CNTs) to alcohol vapours with ultralow power consumption. For the fabrication of the sensor, purified MWNTs were sonicated in $3: 1$ concentrated sulfuric acid and nitric acid for different time intervals. By this method, the MWCNTs can be oxidized and $\mathrm{COOH}$ groups will be grafted along the sidewall and the tube ends of the MWCNTs as shown in Figure 31(a). It is believed that with the polar $\mathrm{COOH}$ groups attached onto the nanotubes surface, the sensors will give stronger responses towards the alcohol vapours as their absorption efficiency with these volatile organic molecules will be increased due to the dipole-dipole interactions (mainly hydrogen bonding) between the $\mathrm{COOH}$ and the polar organic molecules. Figure 31(b) shows a schematic diagram of how the ethanol 


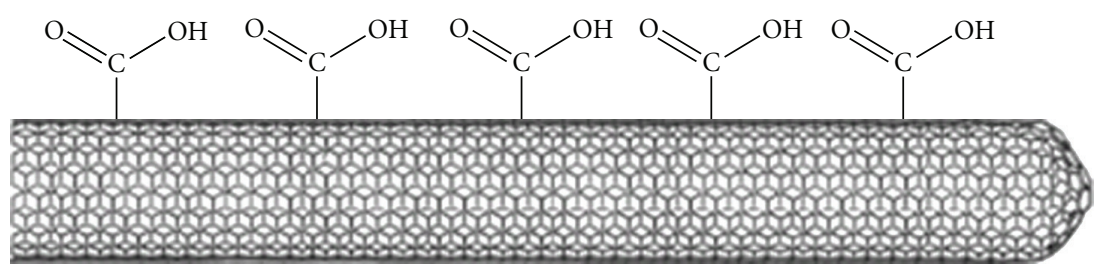

(a)

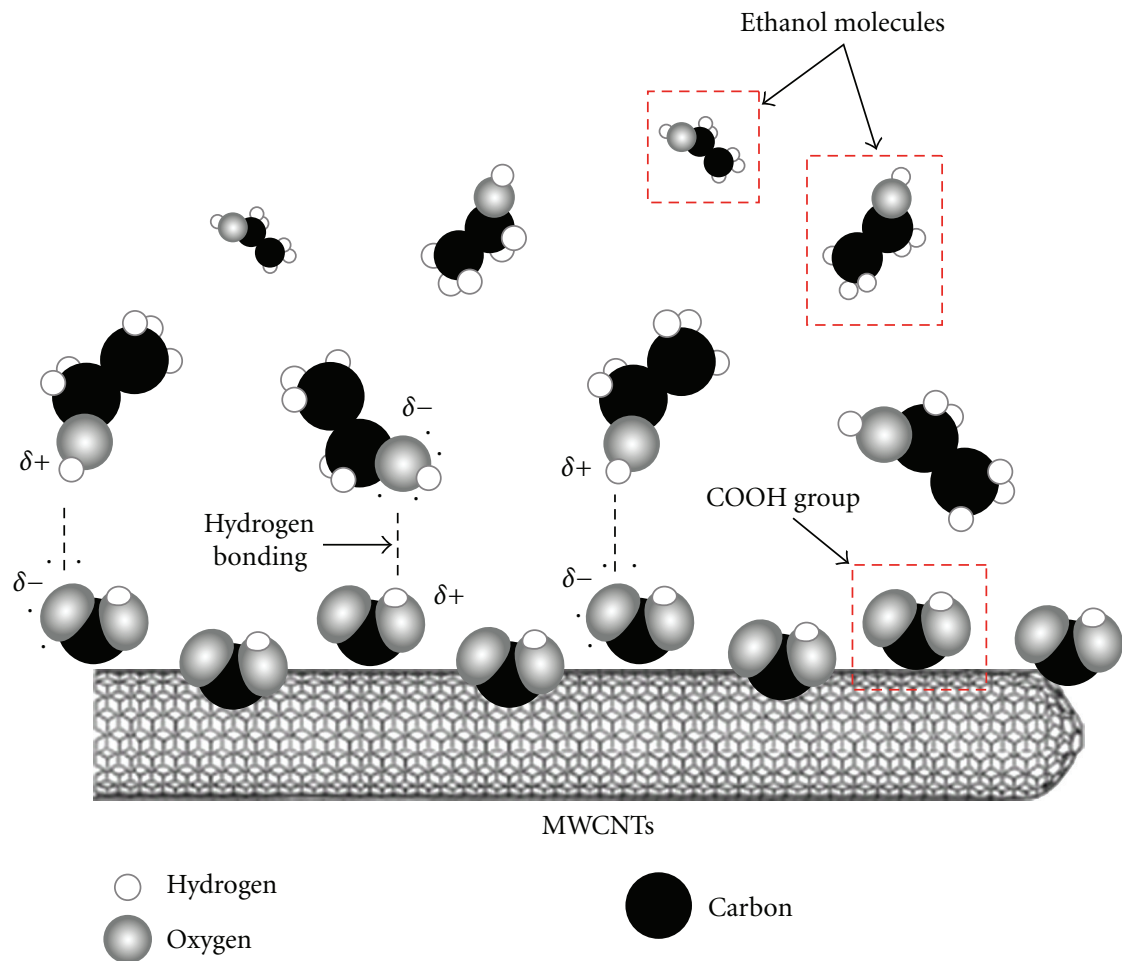

(b)

FIGURE 31: (a) Schematic diagram of the chemically functionalized carbon nanotubes, which have COOH groups attached along the sidewall of the MWCNTs. (b) The proposed mechanism for alcohol vapour detection using f-CNTs sensors. The COOH groups tend to form hydrogen bonding with the ethanol molecules at room temperature [72].

molecules interact with the $\mathrm{COOH}$ groups through hydrogen bonds. The f-CNTs were batch manipulated across Au microelectrodes by electrophoresis and their resistance change was measured for alcohol exposure. The sensor showed a linear response to alcohol vapour concentrations from 1 to $21 \mathrm{ppm}$ and good selectivity to air flow, water vapour and alcohol vapour. Compared to bare CNTs, the functionalized CNTs increased the sensitivity from $\sim 0.9 \%$ to $9.6 \%$.

Hydrogen sensing is always an important topic in gas sensing research due to the safety concerns of handling hydrogen-based utilities. Although CNTs have been proved to be a good gas sensing material, pure CNTs show no response to $\mathrm{H}_{2}$ due to the week binding energy [73]. However, when functionalized with Pt or Pd, which act as a catalyst for the adsorption of $\mathrm{H}_{2}, \mathrm{CNT}$ can be very sensitive to hydrogen [74, 75]. Take $\mathrm{Pt}$ as an example, hydrogen molecules dissociate into atomic hydrogen on Pt surfaces and form $\mathrm{PtH} 2$, which will lower the work function of $\mathrm{Pt}$ and cause the electron transfer from Pt to MWNTs [76-78].
There are several ways to functionalize CNTs with $\mathrm{Pt}$ or Pd. Krishna Kumar and Ramaprabhu [79] dispersed Pt nanoparticles into MWNTs by a series of chemical solution treatment. Suehiro demonstrated two effective methods [80]. For one method, SWCNTs were DEP-trapped onto a microelectrode made of $\mathrm{Pd}$ so that the CNT/Pd interface was formed at both ends of CNTs lying over the Pd electrode surface (shown in Figure 32(a)). The other type of CNT/Pd gas sensor was fabricated by simultaneously DEP-trapping SWCNTs and $\mathrm{Pd}$ nanoparticles onto a chromium $(\mathrm{Cr})$ microelectrode (see Figure 32(b)). Both types of the CNT/Pd gas sensors could respond to hydrogen, while the CNT sensor without the Pd functionalization could not.

5.2. CNTs/Polymer Composites Gas Sensors. Organic polymers are one of the principal materials applied in gas sensing systems. Some conducting polymers can behave like semiconductors due to their heterocyclic compounds which display physicochemical characteristics. As a result, 


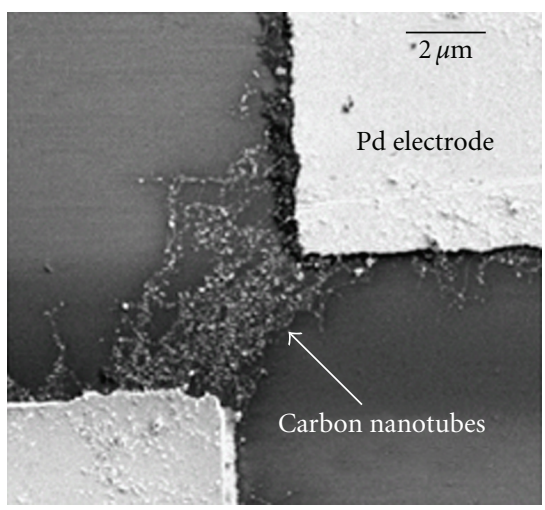

(a)

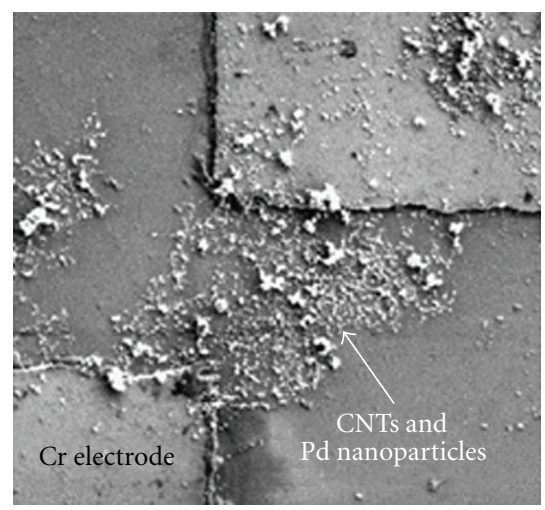

(b)

FIGURE 32: Scanning electron microscope (SEM) images of types A and B sensors trapped in castellated microelectrode gaps by positive DEP: (a) type A (CNTs trapped onto a Pd microelectrode); (b) type $\mathrm{B}$ (CNTs and Pd nanoparticles trapped onto a $\mathrm{Cr}$ microelectrode) [80].

reversible changes in the sensing layer's conductivity can be detected upon polar chemicals' adsorption on the surfaces at room temperature [81]. This effect is believed to be caused by the charge transfer between gas molecules and the polymer or the polymer film's swelling [82]. This sensing response has intensively driven the motivation to develop high sensitive and selective gas/chemical sensors by tailoring the compounds of different organic polymers.

Non-conductive polymers can also be used as gas sensors. For some polymers, the bulk dissolution of gas into the film can cause changes of their physical properties [82]. Since most physical property changes are not readily detectable; researchers have tried to integrate polymers with other functional sensing elements to achieve easier detection process. For example, a recent experimental and theoretical study on the chemical sensing characteristic of polymercoated resonators was reported by Avramov et al. [83] and Yantchev et al. [84].

Another promising sensing material based on polymers is conducting particles-insulating matrix composition. The polymer acts as the insulating matrix while dispersed conducting particles provide the conducting path for sensing

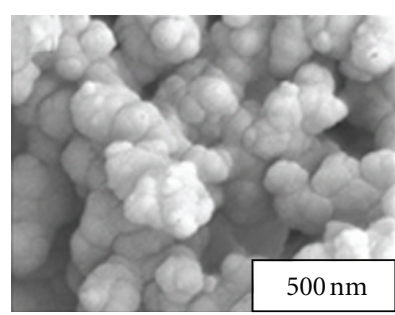

(a)

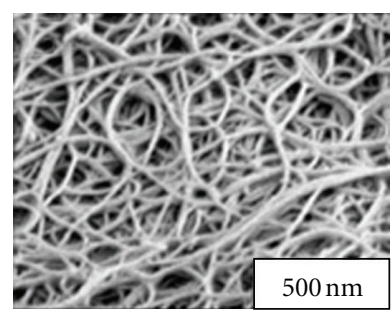

(b)

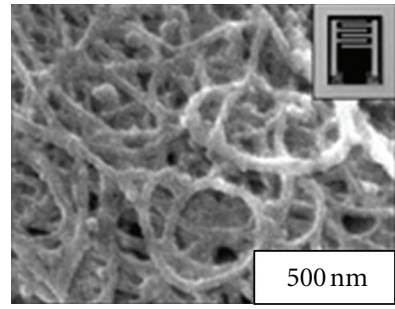

(c)

Figure 33: FESEM images of (a) pure Ppy, (b) purified SWNT bundles, and (c) the SWNT/Ppy nanocomposite. Inset: optical microscope image of the electrodes [90].

[85]. Due to adsorption of interested analyst, there are volumetric changes of the matrix polymer. This can lead to a distinct change in percolation-type conductivity around a critical composition of the material, which is known as "percolation threshold". Generally, the percolation threshold is dependent on the shape of the conducting particle. Composite consisted of particles with higher aspect ratio shows lower threshold and higher sensitivity [86]. CNTs, with almost one-dimensional thread-like structure and good conductivity, are ideal as the dispersed particles in this conducting particles-insulating matrix composition for gas sensing systems. Therefore, CNTs/polymer composites have been intensively studied for gas sensors [87-89].

An et al. [90] prepared an SWNT/polypyrrole (Ppy) nanocomposite by a simple and straightforward in situ chemical polymerization of pyrrole and SWMTs mixture. The composite was then spin-casted onto prepatterned IDEs for resistance measurement. Figure 33 shows the SEM image of the Ppy, SWNTs, and SWNT/Ppy nanocomposite. The resistance responses of all three materials to $\mathrm{NO}_{2}$ are shown in Figure 34. Although all of them showed degradation under long time exposure, the nanocompite showed a better sensitivity than the pure materials, which was due to the enhanced conductivity of the material by dispersed CNTs. 


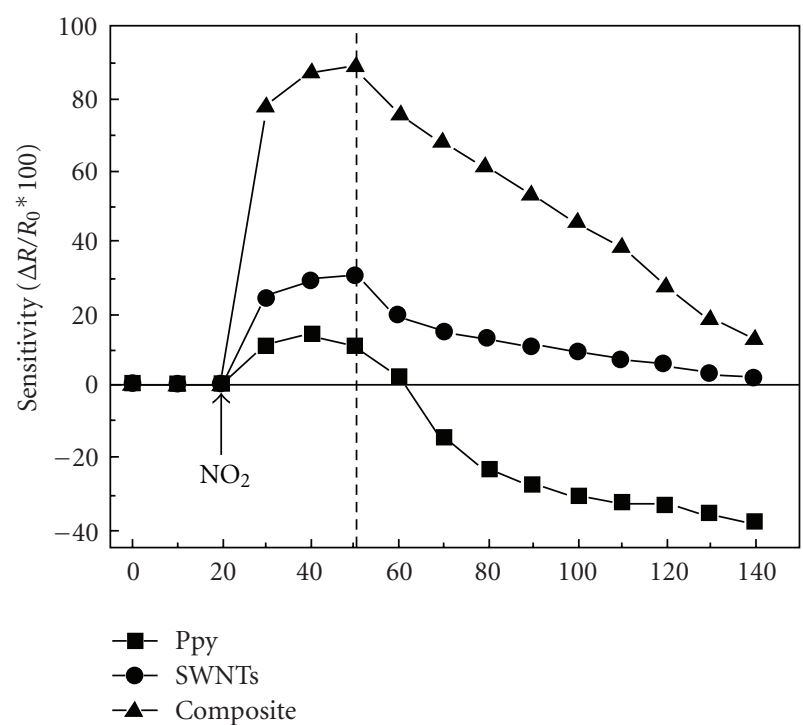

FIgURe 34: The change in sensitivity as a function of gas exposure time at an $\mathrm{NO}_{2}$ concentration of $3000 \mathrm{ppm}$ [90]

Cho et al. investigated the chemical sensing characteristic of SWCNT-Ethyl cellulose (EC) composite [85]. To get uniform dispersion of CNTs and stable composite, the material was prepared carefully with several steps. Poly ( $\mathrm{p}$-phenylenevinylene-co-2,5-dioctoxy-mphenylenevinylene) (PmPV) was used as the dispersant of the nanotubes and chloroform was used as the media. The nanotubes sol was sonicated for 4 hours before mixed with EC, and then the mixture was sonicated for other 6 hours. The mixed sol were sprayed on interdigitated electrodes and annealed at $80^{\circ} \mathrm{C}$ for 24 hours to form the thin sensing film. The sensor's response to benzene and ethanol was measured separately as a function of gas concentration and SWCNTs' content in the composite. The change of resistance showed almost linear relationship with concentration. The maximum sensitivity was $9 \%$ for the film with $5 \mathrm{wt} . \%$ SWCNT contents when exposured to 5,000 ppm of benzene. The sensor's response to ethanol was less obvious and with some noise, however, it showed good linearity within the concerned range with short response and recovery time, as shown in Figure 35.

Based on the same idea, Philip et al. [91] developed composite thin film of polymethylmethacrylate (PMMA) with MWCNTs or oxidation-modified MWCNTs (f-CNTs) for gas sensing. The resistance changes of both the composites were evaluated upon exposure to different gases including dichloromethane, chloroform, acetone, and so forth. Both the CNT/PMMA and the f-CNT/PMMA composites showed increasing resistance upon exposure to these vapours at room temperature, as shown in Figure 36. This behaviour was explained on the basis of volume expansion and polar interaction of the CNT surface with vapour molecules. The f-CNT/PMMA showed significant improvement on the sensor's behaviour including the sensitivity and the response time and recovery, as shown in Figure 36 . This can be

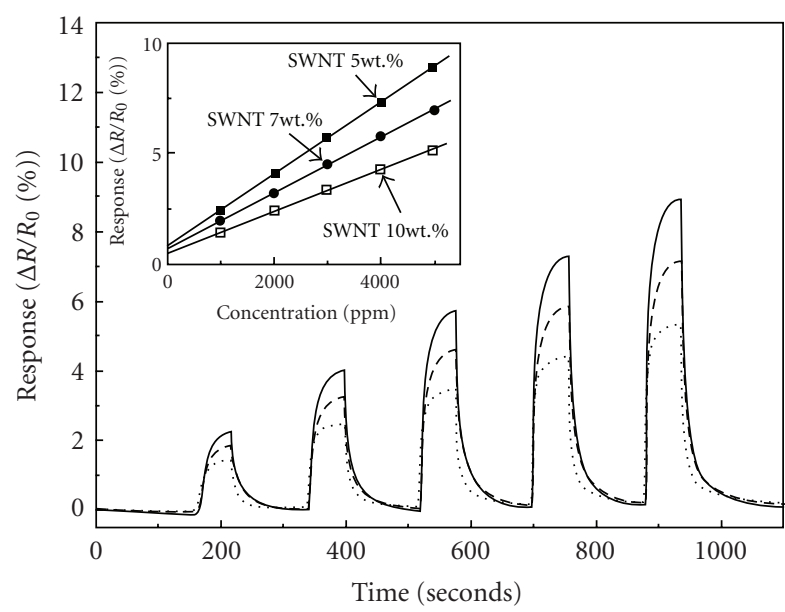

SWNT-EC composite Analyte: benzene

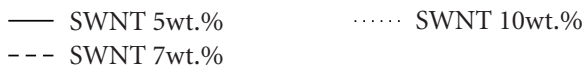

(a)

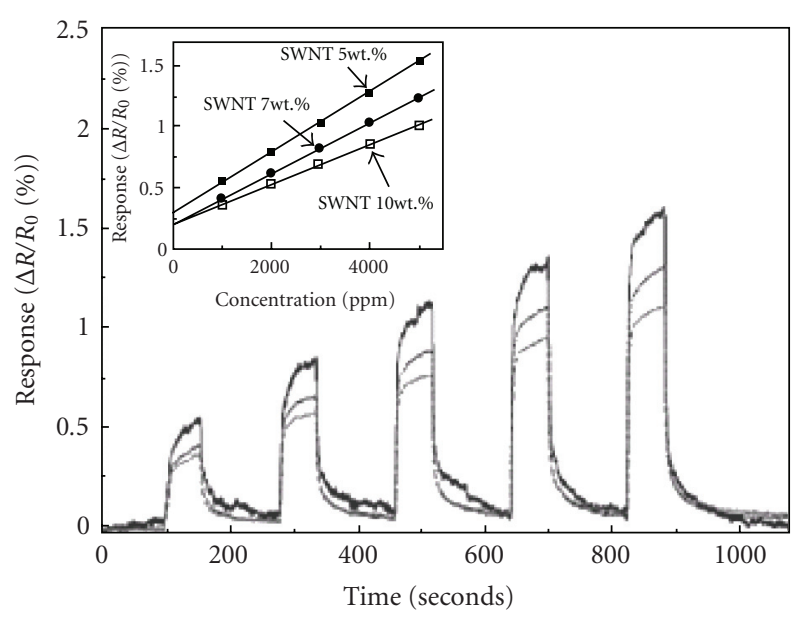

SWNT-EC composite Analyte: ethanol

- SWNT 5wt.\% SWNT 7wt.\%

- - SWNT 10wt. $\%$

(b)

Figure 35: (a) The gas sensing properties of the SWNT-Polymer (EC) composites for benzene vapour. (b) The gas sensing properties of the SWNT-Polymer (EC) composites for ethanol vapour. The concentrations of the vapours ranged from $1000 \mathrm{ppm}$ to $5000 \mathrm{ppm}$. The insets in the upper left corners show the linear relationship [85].

explained by the effects of oxidation on the electronic properties of CNTs that was discussed in a previous section.

In addition of $\mathrm{CNT} /$ polymer composite, the sensing characteristics of polymer-coated CNTs film to specific gases were also investigated. In general, CNTs do not have sensing response to all gases and vapours but only the ones with high adsorption energy or that can interact with them. Therefore, coating or doping of an enhance element on CNTs may broaden the application range. Based on the IDE SWCNTs resistance gas sensor that they developed, 


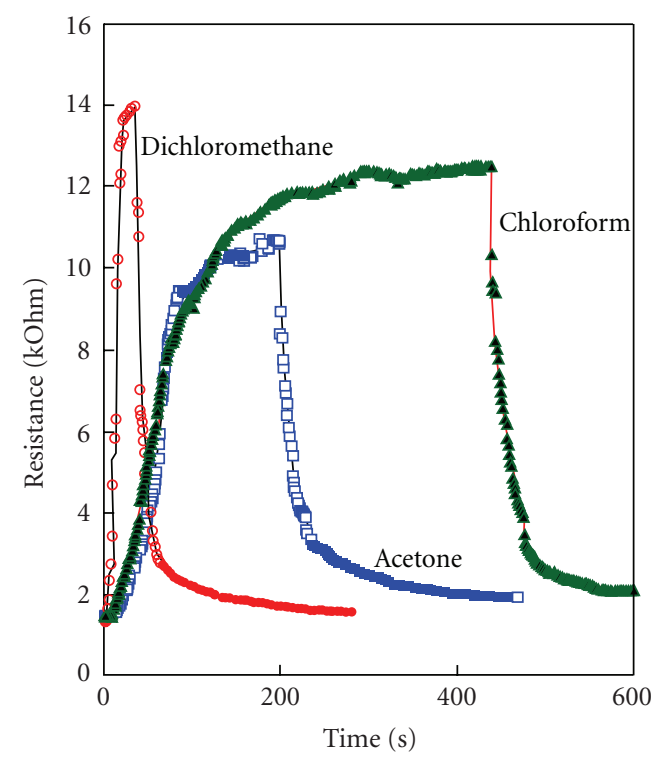

(a)

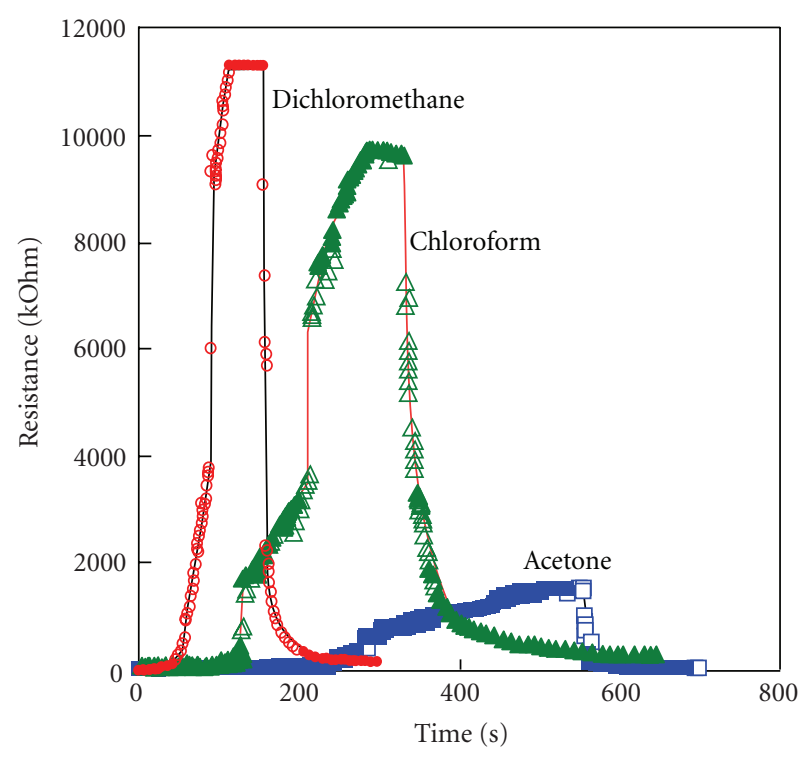

(b)

Figure 36: (a) Response of the CNT/PMMA composite to dichloromethane, chloroform, and acetone vapours; (b) Response of the f-CNT/PMMA composite to dichloromethane, chloroform and acetone vapours [91].

Li et al. studied the sensing character of the same structure but with polymer coatings on CNTs [92]. Chlorosulfonated polyethylene was dissolved in tetrahydrofuran (THF) solvent as a coating solution for $\mathrm{Cl}_{2}$ sensing, and Hydroxypropyl cellulose was dissolved in chloroform as coating to detect $\mathrm{HCl}$. The resistance of polymer coated SWCNT film changed upon exposure to $\mathrm{Cl}_{2}$ and $\mathrm{HCl}$ while uncoated pure SWCNTs showed no sensing signal. The sensing response demonstrates a huge potential of using modified CNTs gas sensing materials for a broad range of gases and chemical vapours.

\section{Conclusions}

With less than twenty years history, the progress and application in CNTs-related technology has been developed impressively fast due to their unique inherent morphologies and properties. CNTs-based sensors have demonstrated huge potential and found their application in various areas. Gas sensors based on both SWNTs and MWNTs have been developed. The nanotubes can be integrated with different sensor architectures by direct-growing, drop-deposition, printing, or DEP method. It has been proved both theoretically and experimentally that, the electrical resistance, thermoelectric power, and local density of states of SWNTs can be reversibly changed by exposure to certain vapours. This can be attributed to the charge transfer between the molecules and the nanotubes, or the change of the charge carries lifetime in CNTs. Therefore, gas sensing FET or resistors that measure the resistance change of CNTs as the transducer is the most commonly used sensor structures. However, this limits the range of the gases that CNTs sensors can detect, especially for the sensing of inert agents. Compared with the resistance sensors, CNTs enhanced ionization chambers allow the detection of gas molecules with low adsorption energy, hence a wide range of vapours. With CNTs integrated, the breakdown voltage can be lowered significantly. Other promising methods to improve the sensing characteristics include the functionalization of CNTs and nanocomposites of CNTs with various polymers, which can broaden the sensing range or enhance the sensitivity. CNTs can also be integrated into resonators sensors. By measuring the change of the resonate frequency, wireless gas sensing can be achieved.

Although CNTs have demonstrated their great potential for gas sensing experimentally, there are still several challenges remained before the real-world application. Synthesise of pure and ideal CNTs is still challenging and costly. It is very difficult to grow defect-free nanotubes continuously to macroscopic length. The precise control over the growth or dispersion of CNTs on surfaces is another problem. Depending on the preparation technique and process, the property and behaviour of the sensors can vary significantly, which is very crucial to the stability of the CNTs-based devices. Therefore, the ability to synthesize of identical and reproducible CNTs with consistent properties is very important for the application of CNTs in all areas. Degradation of the devices has to be investigated more deeply before realworld application. Slow response and recovery is another challenge to be addressed for CNTs-based gas sensors, which is caused by the nature of gas adsorption and desorption process to the nanotubes. However, it was demonstrated that by integrating a microheater under the MWCNTs sensing layer or shortly exposure to UV light, the response time of the sensor can be improved $[93,94]$. It is believed that, with the increase interests and development of related technologies, CNTs gas sensors have a promising future and will bring a huge change to the current industries and our everyday life. 


\section{References}

[1] J. Li, "Carbon nanotubes applications: chemical and physical sensors," in Carbon Nanotubes: Science and Applications, M. Meyyappan, Ed., CRC Press, Boca Raton, Fla, USA.

[2] Z. M. Rittersma, "Recent achievements in miniaturised humidity sensors-a review of transduction techniques," Sensors and Actuators A, vol. 96, no. 2-3, pp. 196-210, 2002.

[3] R. Fenner and E. Zdankiewicz, "Micromachined water vapor sensors: a review of sensing technologies," IEEE Sensors Journal, vol. 1, no. 4, pp. 309-317, 2001.

[4] E. Traversa, "Ceramic sensors for humidity detection: the state-of-the-art and future developments," Sensors and Actuators B, vol. 23, no. 2-3, pp. 135-156, 1995.

[5] S. Iijima, "Helical microtubules of graphitic carbon," Nature, vol. 354, no. 6348, pp. 56-58, 1991.

[6] M. Meyyappan, Carbon Nanotubes: Science and Applications, CRC Press, Boca Raton, Fla, USA.

[7] M. S. Dresselhaus, G. Dresselhaus, and P. C. Eklund, Science of Fullerenes and Carbon Nanotubes, Academic Press, New York, NY, USA, 1996.

[8] R. Saito, M. Fujita, G. Dresselhaus, and M. S. Dresselhaus, "Electronic structure of chiral graphene tubules," Applied Physics Letters, vol. 60, no. 18, pp. 2204-2206, 1992.

[9] M. Dresselhaus, G. Dresselhaus, and P. Avouris, Carbon Nanotubes: Synthesis, Structure, Properties and Applications, Springer, Berlin, Germany, 2001.

[10] N. Sinha, J. Ma, and J. T. W. Yeow, "Carbon nanotube-based sensors," Journal of Nanoscience and Nanotechnology, vol. 6, no. 3, pp. 573-590, 2006.

[11] J. Li and H. T. Ng, "Carbon nanotubes sensors," in Encyclopedia of Nanoscience and Nanotechnology, vol. 1, pp. 591-601, Marcel Dekker, New York, NY, USA, 2004.

[12] H. Dai, "Nanotube growth and characterization," in Carbon Nanotubes: Synthesis, Structure, Properties and Applications, M. S. Dresselhaus and G. Dresselhaus, Eds., pp. 29-54, Springer, New York, NY, USA, 2000.

[13] T. W. Ebbesen, "Production and purification of carbon nanotubes," in Carbon Nanotubes: Preparation and Properties, T. W. Ebbesen, Ed., pp. 139-162, CRC Press, Boca Raton, Fla, USA, 1997.

[14] J. Li, Y. Lu, Q. Ye, et al., "Carbon nanotubes sensors for gas and organic vapour detection," Nano Letters, vol. 3, no. 7, pp. 929-933, 2003.

[15] Y. D. Lee, W.-S. Cho, S.-I. Moon, et al., "Gas sensing properties of printed multiwalled carbon nanotubes using the field emission effect," Chemical Physics Letters, vol. 433, no. 1-3, pp. 105-109, 2006.

[16] K. Yamamoto, S. Akita, and Y. Nakayama, "Orientation and purification of carbon nanotubes using ac electrophoresis," Journal of Physics D, vol. 31, no. 8, pp. L34-L36, 1998.

[17] K. Bubke, H. Gnewuch, M. Hempstead, J. Hammer, and M. L. H. Green, "Optical anisotropy of dispersed carbon nanotubes induced by an electric field," Applied Physics Letters, vol. 71, no. 14, pp. 1906-1908, 1997.

[18] T. Prasse, J.-Y. Cavaille, and W. Bauhofer, "Electric anisotropy of carbon nanofibre/epoxy resin composites due to electric field induced alignment," Composites Science and Technology, vol. 63, no. 13, pp. 1835-1841, 2003.

[19] X. Q. Chen, T. Saito, H. Yamada, and K. Matsushige, "Aligning single-wall carbon nanotubes with an alternating-current electric field," Applied Physics Letters, vol. 78, no. 23, pp. 37143716, 2001.
[20] X. Liu, J. L. Spencer, A. B. Kaiser, and W. M. Arnold, "Electricfield oriented carbon nanotubes in different dielectric solvents," Current Applied Physics, vol. 4, no. 2-4, pp. 125-128, 2004.

[21] F. Wakaya, T. Nagai, and K. Gamo, "Position control of carbon nanotube using patterned electrode and electric field," Microelectronic Engineering, vol. 63, no. 1-3, pp. 27-31, 2002.

[22] R. Krupke, F. Hennrich, H. B. Weber, et al., "Contacting single bundles of carbon nanotubes with alternating electric fields," Applied Physics A, vol. 76, no. 3, pp. 397-400, 2003.

[23] J. Suehiro, G. Zhou, and M. Hara, "Fabrication of a carbon nanotube-based gas sensor using dielectrophoresis and its application for ammonia detection by impedance spectroscopy," Journal of Physics D, vol. 36, no. 21, pp. L109L114, 2003.

[24] J. Suehiro, H. Imakiire, S. Hidaka, et al., "Schottky-type response of carbon nanotube $\mathrm{NO}_{2}$ gas sensor fabricated onto aluminum electrodes by dielectrophoresis," Sensors and Actuators B, vol. 114, no. 2, pp. 943-949, 2006.

[25] C. S. Huang, B. R. Huang, Y. H. Jang, M. S. Tsai, and C. Y. Yeh, "Three-terminal CNTs gas sensor for $\mathrm{N}_{2}$ detection," Diamond and Related Materials, vol. 14, no. 11-12, pp. 1872-1875, 2005.

[26] Y.-T. Jang, S.-I. Moon, J.-H. Ahn, Y.-H. Lee, and B.-K. Ju, “A simple approach in fabricating chemical sensor using laterally grown multi-walled carbon nanotubes," Sensors and Actuators $B$, vol. 99, no. 1, pp. 118-122, 2004.

[27] J. Zhao, A. Buldum, J. Han, and J. P. Lu, "Gas molecule adsorption in carbon nanotubes and nanotube bundles," Nanotechnology, vol. 13, no. 2, pp. 195-200, 2002.

[28] M. Tabib-Azar and Y. Xie, "Sensitive $\mathrm{NH}_{3} \mathrm{OH}$ and $\mathrm{HCl}$ gas sensors using self-aligned and self-welded multiwalled carbon nanotubes," IEEE Sensors Journal, vol. 7, no. 10, pp. 14351439, 2007.

[29] D. Ding, Z. Chen, S. Rajaputra, and V. Singh, "Hydrogen sensors based on aligned carbon nanotubes in an anodic aluminum oxide template with palladium as a top electrode," Sensors and Actuators B, vol. 124, no. 1, pp. 12-17, 2007.

[30] T. Ueda, S. Katsuki, and N. Heidari Abhari, "Effect of laser irradiation on carbon nanotube films for $\mathrm{NO}_{x}$ gas sensor," Surface \& Coatings Technology, vol. 202, pp. 5325-5328, 2008.

[31] S. Peng and K. Cho, "Chemical control of nanotube electronics," Nanotechnology, vol. 11, no. 2, pp. 57-60, 2000.

[32] C. Cantalini, L. Valentini, L. Lozzi, I. Armentano, J. M. Kenny, and S. Santucci, " $\mathrm{NO}_{2}$ gas sensitivity of carbon nanotubes obtained by plasma enhanced chemical vapor deposition," Sensors and Actuators B, vol. 93, no. 1-3, pp. 333-337, 2003.

[33] P. G. Collins, K. Bradley, M. Ishigami, and A. Zettl, "Extreme oxygen sensitivity of electronic properties of carbon nanotubes," Science, vol. 287, no. 5459, pp. 1801-1804, 2000.

[34] K. A. Williams and P. C. Eklund, "Monte Carlo simulations of $\mathrm{H}_{2}$ physisorption in finite-diameter carbon nanotube ropes," Chemical Physics Letters, vol. 320, no. 3-4, pp. 352-358, 2000.

[35] G. Stan and M. W. Cole, "Hydrogen adsorption in nanotubes," Journal of Low Temperature Physics, vol. 110, no. 1-2, pp. 539$544,1998$.

[36] C. K. W. Adu, G. U. Sumanasekera, B. K. Pradhan, H. E. Romero, and P. C. Eklund, "Carbon nanotubes: a thermoelectric nano-nose," Chemical Physics Letters, vol. 337, no. 1-3, pp. 31-35, 2001.

[37] J. Han, in Carbon Nanotubes: Science and Application, M. Meyyappan, Ed., CRC Press LLC, Boca Raton, Fla, USA, 2005.

[38] G. U. Sumanasekera, C. K. W. Adu, S. Fang, and P. C. Eklund, "Effects of gas adsorption and collisions on electrical transport in single-walled carbon nanotubes," Physical Review Letters, vol. 85, no. 5, pp. 1096-1099, 2000. 
[39] X.-P. Tang, A. Kleinhammes, H. Shimoda, et al., "Electronic structures of single-walled carbon nanotubes determined by NMR," Science, vol. 288, no. 5465, pp. 492-494, 2000.

[40] S.-H. Jhi, S. G. Louie, and M. L. Cohen, "Electronic properties of oxidized carbon nanotubes," Physical Review Letters, vol. 85, no. 8 , pp. 1710-1713, 2000.

[41] R. D. Barnard, Thermoelectricity in Metals and Alloys, John Wiley \& Sons, New York, NY, USA, 1972.

[42] H. E. Romero, G. U. Sumanasekera, S. Kishore, and P. C. Eklund, "Effects of adsorption of alcohol and water on the electrical transport of carbon nanotube bundles," Journal of Physics: Condensed Matter, vol. 16, no. 12, pp. 1939-1949, 2004.

[43] S. Peng and K. Cho, "Ab initio study of doped carbon nanotube sensors," Nano Letters, vol. 3, no. 4, pp. 513-517, 2003.

[44] Z. Weng-Sieh, K. Cherrey, N. G. Chopra, et al., "Synthesis of BxCyNz nanotubules," Physical Review B, vol. 51, no. 16, pp. 11229-11232, 1995.

[45] W.-Q. Han, J. Cumings, X. Huang, K. Bradley, and A. Zettl, "Synthesis of aligned $\mathrm{BxCyNz}$ nanotubes by a substitutionreaction route," Chemical Physics Letters, vol. 346, no. 5-6, pp. 368-372, 2001.

[46] J. Kong, N. R. Franklin, C. Zhou, et al., "Nanotube molecular wires as chemical sensors," Science, vol. 287, no. 5453, pp. 622 $625,2000$.

[47] T. Someya, J. Small, P. Kim, C. Nuckolls, and J. T. Yardley, "Alcohol vapor sensors based on single-walled carbon nanotube field effect transistors," Nano Letters, vol. 3, no. 7, pp. 877-881, 2003.

[48] O. K. Varghese, P. D. Kichambre, D. Gong, K. G. Ong, E. C. Dickey, and C. A. Grimes, "Gas sensing characteristics of multi-wall carbon nanotubes," Sensors and Actuators B, vol. 81, no. 1, pp. 32-41, 2001.

[49] J. Chung, K.-H. Lee, J. Lee, D. Troya, and G. C. Schatz, "Multiwalled carbon nanotubes experiencing electrical breakdown as gas sensors," Nanotechnology, vol. 15, no. 11, pp. 1596-1602, 2004.

[50] J. Sippel-Oakley, H. T. Wang, B. S. Kang, et al., "Carbon nanotube films for room temperature hydrogen sensing," Nanotechnology, vol. 16, pp. 2218-2221, 2005.

[51] T. Ueda, S. Katsuki, K. Takahashi, H. A. Narges, T. Ikegami, and F. Mitsugi, "Fabrication and characterization of carbon nanotube based high sensitive gas sensors operable at room temperature," Diamond and Related Materials, vol. 17, no. 710, pp. 1586-1589, 2008.

[52] L. Valentini, C. Cantalini, I. Armentano, J. M. Kenny, L. Lozzi, and S. Santucci, "Highly sensitive and selective sensors based on carbon nanotubes thin films for molecular detection," Diamond and Related Materials, vol. 13, no. 4-8, pp. 13011305, 2004.

[53] Y. M. Wong, W. P. Kang, J. L. Davidson, A. Wisitsora-At, and K. L. Soh, "A novel microelectronic gas sensor utilizing carbon nanotubes for hydrogen gas detection," Sensors and Actuators B, vol. 93, no. 1-3, pp. 327-332, 2003.

[54] Z. Hou, D. Xu, and B. Cai, "Ionization gas sensing in a microelectrode system with carbon nanotubes," Applied Physics Letters, vol. 89, no. 21, Article ID 213502, 3 pages, 2006.

[55] A. Modi, N. Koratkar, E. Lass, B. Wei, and P. M. Ajayan, "Miniaturized gas ionization sensors using carbon nanotubes," Nature, vol. 424, no. 6945, pp. 171-174, 2003.

[56] W. A. De Heer, A. Chatelain, and D. Ugarte, "A carbon nanotube field-emission electron source," Science, vol. 270, no. 5239, pp. 1179-1180, 1995.
[57] N. de Jonge, Y. Lamy, K. Schoots, and T. H. Oosterkamp, "High brightness electron beam from a multi-walled carbon nanotube," Nature, vol. 420, no. 6914, pp. 393-395, 2002.

[58] S. J. Kim, "Gas sensors based on Paschen's law using carbon nanotubes as electron emitters," Journal of Physics D, vol. 39, no. 14, pp. 3026-3029, 2006.

[59] S. Kim, "CNT sensors for detecting gases with low adsorption energy by ionization," Sensors, vol. 6, no. 5, pp. 503-513, 2006.

[60] J. Wu, H. Liu, D. Xu, et al., in Proceedings of the $3 \mathrm{rd}$ IEEE InternationalConference on Nano/Micro Engineering and Molecular System, Sanya, China, January 2008.

[61] A. Wadhawan, R. E. Stallcup II, K. F. Stephens II, J. M. Perez, and I. A. Akwani, "Effects of $\mathrm{O}_{2}, \mathrm{Ar}$, and $\mathrm{H}_{2}$ gases on the fieldemission properties of single-walled and multiwalled carbon nanotubes," Applied Physics Letters, vol. 79, no. 12, pp. 18671869, 2001.

[62] Z. Yong, L. Junhua, and L. Xin, "The structure optimization of the carbon nanotube film cathode in the application of gas sensor," Sensors and Actuators A, vol. 128, pp. 278-289, 2006.

[63] J. T. W. Yeow and J. P. M. She, "Carbon nanotube-enhanced capillary condensation for a capacitive humidity sensor," Nanotechnology, vol. 17, no. 21, pp. 5441-5448, 2006.

[64] E. S. Snow, F. K. Perkins, E. J. Houser, S. C. Badescu, and T. L. Reinecke, "Chemical detection with a single-walled carbon nanotube capacitor," Science, vol. 307, no. 5717, pp. 19421945, 2005.

[65] S. Chopra, A. Pham, J. Gaillard, A. Parker, and A. M. Rao, "Carbon-nanotube-based resonant-circuit sensor for ammonia," Applied Physics Letters, vol. 80, no. 24, pp. 46324636, 2002.

[66] S. Chopra, K. McGuire, N. Gothard, A. M. Rao, and A. Pham, "Selective gas detection using a carbon nanotube sensor," Applied Physics Letters, vol. 83, no. 11, pp. 2280-2282, 2003.

[67] M. Penza, F. Antolini, and M. Vittori Antisari, "Carbon nanotubes as SAW chemical sensors materials," Sensors and Actuators B, vol. 100, no. 1-2, pp. 47-59, 2004.

[68] K. G. Ong, K. Zeng, and C. A. Grimes, "A wireless, passive carbon nanotube-based gas sensor," IEEE Sensors Journal, vol. 2, no. 2, pp. 82-88, 2002.

[69] J. L. Bahr and J. M. Tour, "Covalent chemistry of single-wall carbon nanotubes," Journal of Materials Chemistry, vol. 12, no. 7, pp. 1952-1958, 2002.

[70] A. Hirsch, "Functionalization of single-walled carbon nanotubes," Angewandte Chemie International Edition, vol. 41, no. 11, pp. 1853-1859, 2002.

[71] S. Niyogi, M. A. Hamon, H. Hu, et al., "Chemistry of singlewalled carbon nanotubes," Accounts of Chemical Research, vol. 35, no. 12, pp. 1105-1113, 2002.

[72] M. L. Y. Sin, G. C. T. Chow, G. M. K. Wong, W. J. Li, P. H. W. Leong, and K. W. Wong, "Ultralow-power alcohol vapor sensors using chemically functionalized multiwalled carbon nanotubes," IEEE Transactions on Nanotechnology, vol. 6, no. 5, pp. 571-577, 2007.

[73] S. Dag, Y. Ozturk, S. Ciraci, and T. Yildirim, "Adsorption and dissociation of hydrogen molecules on bare and functionalized carbon nanotubes," Physical Review B, vol. 72, no. 15, Article ID 155404, 8 pages, 2005.

[74] I. Sayago, E. Terrado, E. Lafuente, et al., "Hydrogen sensors based on carbon nanotubes thin films," Synthetic Metals, vol. 148, no. 1, pp. 15-19, 2005.

[75] J. Kong, M. G. Chapline, and H. Dai, "Functionalized carbon nanotubes for molecular hydrogen sensors," Advanced Materials, vol. 13, no. 18, pp. 1384-1386, 2001. 
[76] A. T. Gee, B. E. Hayden, C. Mormiche, and T. S. Nunney, "The role of steps in the dynamics of hydrogen dissociation on $\operatorname{Pt}(533)$," Journal of Chemical Physics, vol. 112, no. 17, pp. 7660-7668, 2000.

[77] R. A. Olsen, Ş. C. Bǎdescu, S. C. Ying, and E. J. Baerends, "Adsorption and diffusion on a stepped surface: atomic hydrogen on Pt(211)," Journal of Chemical Physics, vol. 120, no. 24, pp. 11852-11863, 2004.

[78] A. Mandelis and C. Christofides, Physics, Chemistry and Technology of Solid State Gas Sensor Devices, John Wiley \& Sons, New York, NY, USA, 1993.

[79] M. Krishna Kumar and S. Ramaprabhu, "Nanostructured Pt functionlized multiwalled carbon nanotube based hydrogen sensor," Journal of Physical Chemistry B, vol. 110, no. 23, pp. 11291-11298, 2006.

[80] J. Suehiro, S. Hidaka, S. Yamane, and K. Imasaka, "Fabrication of interfaces between carbon nanotubes and catalytic palladium using dielectrophoresis and its application to hydrogen gas sensor," Sensors and Actuators B, vol. 127, no. 2, pp. 505$511,2007$.

[81] P. N. Bartlett, P. B. M. Archer, and S. K. Ling-Chung, "Conducting polymer gas sensors-part I: fabrication and characterization," Sensors and Actuators, vol. 19, no. 2, pp. 125-140, 1989.

[82] M. C. Petty and R. Casalini, "Gas sensing for the 21st century: the case for organic thin films," Engineering Science and Education Journal, vol. 10, no. 3, pp. 99-105, 2001.

[83] I. D. Avramov, S. Kurosawa, M. Rapp, P. Krawczak, and E. I. Radeva, "Investigations on plasma-polymer-coated SAW and STW resonators for chemical gas-sensing applications," IEEE Transactions on Microwave Theory and Techniques, vol. 49, no. 4, pp. 827-837, 2001.

[84] V. M. Yantchev, V. L. Strashilov, M. Rapp, U. Stahl, and I. D. Avramov, "Theoretical and experimental mass-sensitivity analysis of polymer-coated SAW and STW resonators for gas sensing applications," IEEE Sensors Journal, vol. 2, no. 4, pp. 307-312, 2002.

[85] S. M. Cho, Y. J. Kim, Y. S. Kim, Y. Yang, and S.-C. Ha, “The application of carbon nanotube-polymer composite as gas sensing materials," Proceedings of IEEE Sensors, vol. 2, pp. 701704, 2004.

[86] J. K. Abraham, B. Philip, A. Witchurch, V. K. Varadan, and C. Channa Reddy, "A compact wireless gas sensor using a carbon nanotube/PMMA thin film chemiresistor," Smart Materials and Structures, vol. 13, no. 5, pp. 1045-1049, 2004.

[87] Y. Wanna, N. Srisukhumbowornchai, A. Tuantranont, A. Wisitsoraat, N. Thavarungkul, and P. Singjai, "The effect of carbon nanotube dispersion on $\mathrm{CO}$ gas sensing characteristics of polyaniline gas sensor," Journal of Nanoscience and Nanotechnology, vol. 6, no. 12, pp. 3893-3896, 2006.

[88] L. Valentini, V. Bavastrello, E. Stura, I. Armentano, C. Nicolini, and J. M. Kenny, "Sensors for inorganic vapor detection based on carbon nanotubes and poly( $o$-anisidine) nanocomposite material," Chemical Physics Letters, vol. 383, no. 5-6, pp. 617622, 2004.

[89] M. Wienecke, M.-C. Bunescu, M. Pietrzak, K. Deistung, and P. Fedtke, "PTFE membrane electrodes with increased sensitivity for gas sensor applications," Synthetic Metals, vol. 138, no. 1-2, pp. 165-171, 2003.

[90] K. H. An, S. Y. Jeong, H. R. Hwang, and Y. H. Lee, "Enhanced sensitivity of a gas sensor incorporating single-walled carbon nanotube-polypyrrole nanocomposites," Advanced Materials, vol. 16, no. 12, pp. 1005-1009, 2004.
[91] B. Philip, J. K. Abraham, and A. Chandrasekhar, "Carbon nanotube/PMMA composite thin films for gas-sensing applications," Smart Materials and Structures, vol. 12, pp. 935-939, 2003.

[92] J. Li, Y. Lu, and M. Meyyappan, "Nano chemical sensors with polymer-coated carbon nanotubes," IEEE Sensors Journal, vol. 6, no. 5, pp. 1047-1051, 2006.

[93] T. Ueda, M. M. H. Bhuiyan, H. Norimatsu, S. Katsuki, T. Ikegami, and F. Mitsugi, "Development of carbon nanotubebased gas sensors for $\mathrm{NO}_{x}$ gas detection working at low temperature," Physica E, vol. 40, no. 7, pp. 2272-2277, 2008.

[94] W.-S. Cho, S.-I. Moon, Y.-D. Lee, Y.-H. Lee, J.-H. Park, and B. $\mathrm{K}$. Ju, "Multiwall carbon nanotube gas sensor fabricated using thermomechanical structure," IEEE Electron Device Letters, vol. 26, no. 7, pp. 498-500, 2005. 

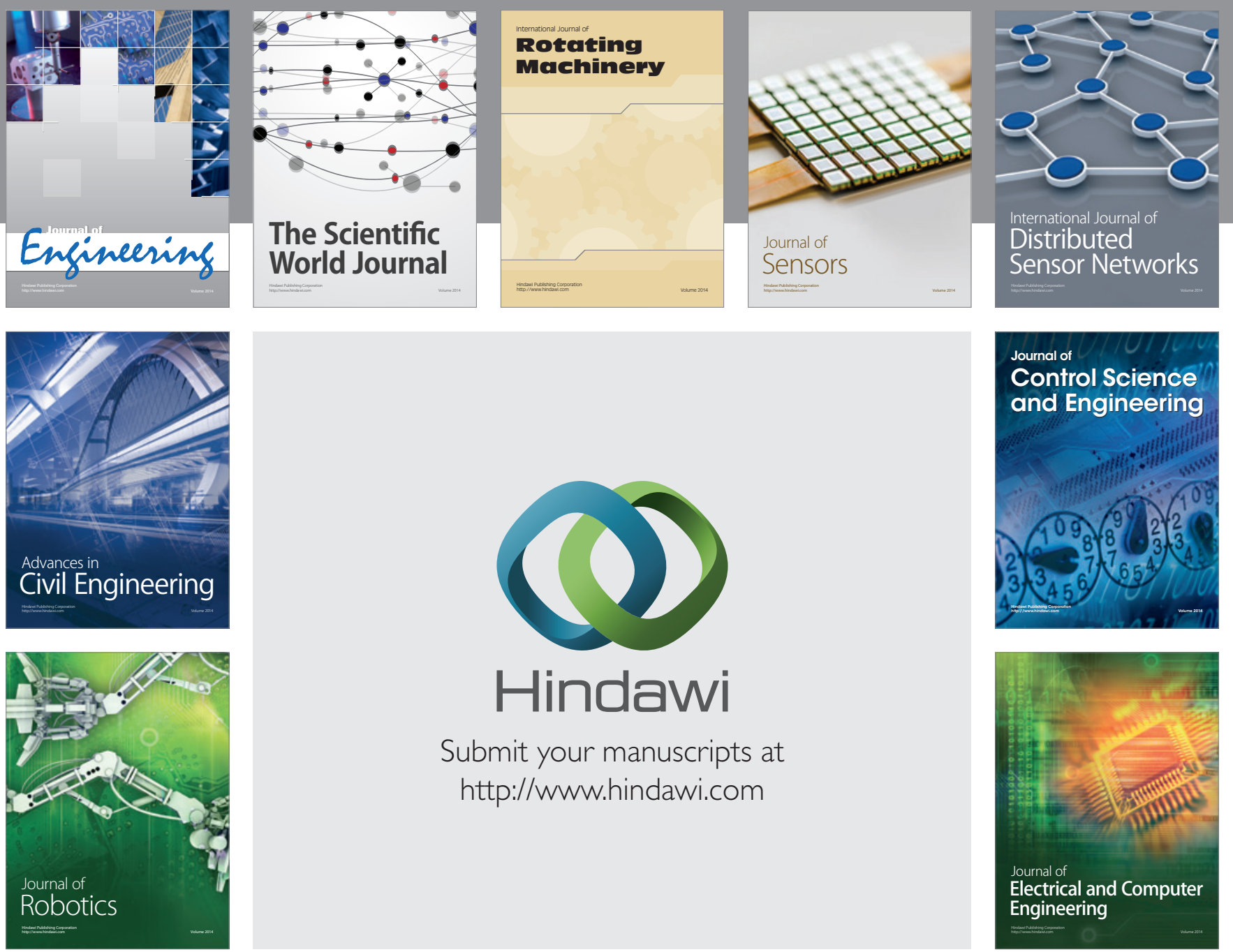

Submit your manuscripts at

http://www.hindawi.com
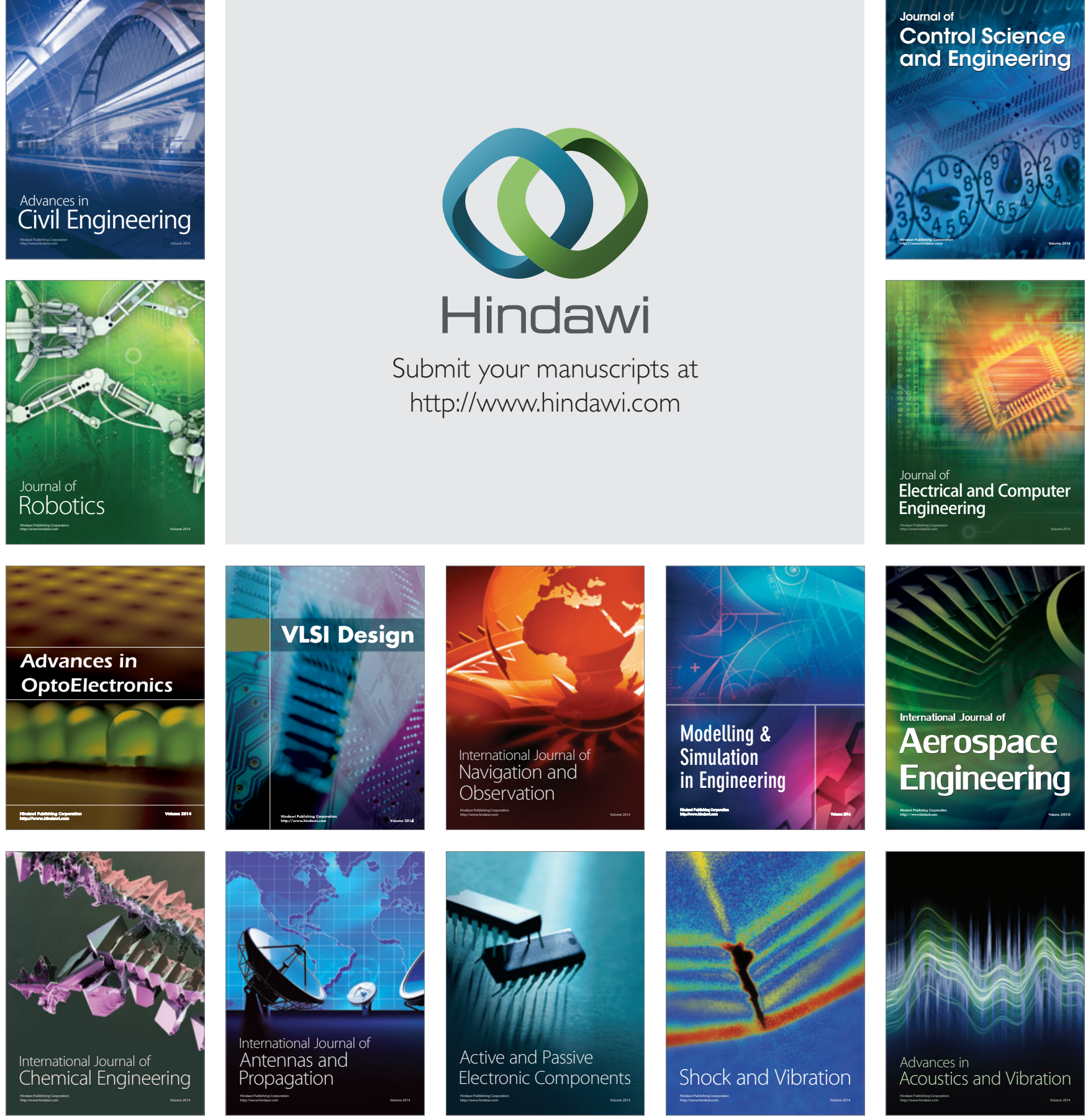